\title{
A $\theta-\gamma$ Oscillation Code for Neuronal Coordination during Motor Behavior
}

\author{
Jun Igarashi, ${ }^{1,2}$ Yoshikazu Isomura, ${ }^{3,4}$ Kensuke Arai, ${ }^{2}$ Rie Harukuni, ${ }^{2}$ and Tomoki Fukai ${ }^{1,2,4}$ \\ ${ }^{1}$ Brain and Neural Systems Team, RIKEN Computational Science Research Program, Saitama 351-0198, Japan, ${ }^{2}$ Laboratory for Neural Circuit Theory, \\ RIKEN Brain Science Institute, Saitama 351-0198, Japan, ${ }^{3}$ Brain Science Institute, Tamagawa University, Tokyo 194-8610, Japan, and ${ }^{4}$ Core Research for \\ Evolutional Science and Technology, Japan Science and Technology Agency, Saitama 332-0012, Japan
}

\begin{abstract}
Sequential motor behavior requires a progression of discrete preparation and execution states. However, the organization of statedependent activity in neuronal ensembles of motor cortex is poorly understood. Here, we recorded neuronal spiking and local field potential activity from rat motor cortex during reward-motivated movement and observed robust behavioral state-dependent coordination between neuronal spiking, $\gamma$ oscillations, and $\theta$ oscillations. Slow and fast $\gamma$ oscillations appeared during distinct movement states and entrained neuronal firing. $\gamma$ oscillations, in turn, were coupled to $\theta$ oscillations, and neurons encoding different behavioral states fired at distinct phases of $\theta$ in a highly layer-dependent manner. These findings indicate that $\theta$ and nested dual band $\gamma$ oscillations serve as the temporal structure for the selection of a conserved set of functional channels in motor cortical layer activity during animal movement. Furthermore, these results also suggest that cross-frequency couplings between oscillatory neuronal ensemble activities are part of the general coding mechanism in cortex.
\end{abstract}

\section{Introduction}

Synchronous neuronal firing is considered to bind cortical neurons into cell assemblies that engage in specific computational functions. Synchronized spikes can exert a strong impact on downstream neurons and hence control the transmission of information between distinct cell assemblies. For example, oscillations in the $\gamma$ band $(30-120 \mathrm{~Hz})$ provide a mechanism to induce synchronized spiking among neurons processing sensory (Womelsdorf and Fries, 2007; Gregoriou et al., 2009), memory (Pesaran et al., 2002; Jutras et al., 2009; Quilichini et al., 2010), and motor information (Murthy and Fetz, 1996; Lee, 2003; Szurhaj and Derambure, 2006). These fast oscillations are sometimes accompanied, or nested hierarchically, by lower-frequency oscillations in the $\theta$ band $(4-10 \mathrm{~Hz})$ in memory-related cortical areas (Quilichini et al., 2010), including hippocampus (Bragin et al., 1995; Lisman and Redish, 2009).

Recently, progress has been made to understand the precise roles of oscillations at different frequencies and their crossfrequency coupling in neural circuit function. Slow $(30-50 \mathrm{~Hz})$ and fast $(60-120 \mathrm{~Hz}) \gamma$ bands coupled to $\theta$ oscillation were

Received May 17, 2013; revised 0ct. 15, 2013; accepted 0ct. 19, 2013.

Author contributions:Y.I. and T.F. designed research; J.I., Y.I., and R.H. performed research; J.I. and K.A. analyzed data; J.I. and T.F. wrote the paper.

This work was supported in part by Grants-in-Aid for Scientific Research on Innovative Areas (no. 22115013) and the Next-Generation Supercomputer Project of MEXT, Japan. We thank Takeshi Kaneko (Kyoto University) for fruitful discussion about the anatomy and electrophysiology of the rat motor cortex.

The authors declare no competing financial interests.

Correspondence should be addressed to Dr. Tomoki Fukai, Lab for Neural Circuit Theory, RIKEN Brain Science Institute, Hirosawa 2-1, Wako, Saitama 351-0198, Japan. E-mail: tfukai@riken.jp.

J. Igarashi's present address: Neural Computation Unit, Okinawa Institute of Science and Technology, 1919-1 Tancha, Onna-son, Kunigami-gun, Okinawa 904-0495, Japan.

DOI:10.1523/JNEUROSCI.2126-13.2013

Copyright $\odot 2013$ the authors $\quad 0270-6474 / 13 / 3318515-16 \$ 15.00 / 0$ shown to entrain hippocampal neurons in phase-locked firing and route the flow of information in hippocampal circuits (Colgin et al., 2009). Distinct roles of different $\gamma$ bands were also proposed in basal ganglia for reward-driven motor behavior (van der Meer et al., 2010) and in neocortex during slow wave sleep (Le Van Quyen et al., 2010). Moreover, oscillation phase coupling was shown to coordinate functional cell assemblies in anatomically dispersed brain areas (Canolty and Knight, 2010; Fujisawa and Buzsáki, 2011).

These findings suggest the potential existence of a conserved neural code coordinating the phase coupling of spiking, $\gamma$ oscillations, and $\theta$ oscillations for brain computation and motivated us to examine whether motor behavior is coordinated by such a hierarchical neural code in neocortical microcircuits. $\gamma$ oscillations are known to appear in the local field potential (LFP) of monkey motor cortex during movement preparation (Sanes and Donoghue, 1993), and sensorimotor cortical neurons fire preferentially near the negative peaks of LFP $\gamma$ oscillations (Murthy and Fetz, 1996; Donoghue et al., 1998). Likewise, the monkey supplementary motor area shows synchronous spiking in the range of slow $\gamma$ (Lee, 2003), and event-related synchronization at $\gamma$ frequencies occurs during execution of motor commands by human subjects (Szurhaj and Derambure, 2006). However, whether and how distinct slow and fast $\gamma$ oscillations differentially coordinate spike firing of motor cortex neurons in relation to stages of motor behavior from preparation to execution has not been studied. Moreover, little is known about whether $\theta$ oscillations have any role in motor ensemble coding. Furthermore, it is also unclear whether $\theta-\gamma$ coupling exists outside of the hippocampal-cortical memory axis and coordinates unit spiking during behavior.

To understand these issues, we analyzed the temporal relationships between the phases of LFP oscillations and spiking of 
morphologically identified pyramidal cells and fast spiking (FS) interneurons recorded juxtacellularly at various depths in the primary motor cortex of behaving rats. We also analyzed the temporal relationship between multiunit spike sequences and LFP oscillations during motor behavior.

\section{Materials and Methods}

Juxtacellular recordings from behaving rats. All experiments were performed in accordance with animal protocols approved by the Experimental Animal Committee of the RIKEN Institute. The results of juxtacellular recordings shown in the present paper were obtained by reanalyzing the raw electrophysiological data recorded previously (Isomura et al., 2009). Here, we briefly summarize the experimental procedure since the details are found in the paper. Adult male Long-Evans rats (150-250 g; Japan SLC) were trained to perform self-paced right forelimb movements (sequence of push, hold, and pull movements of a lever) after we surgically attached a lightweight, custom-made sliding headattachment to the skull of the rats. Then we recorded the activity of neurons in layers 2-6 of the motor cortex juxtacellularly from the headrestrained rats performing the behavioral task. After recording of taskrelated spike activity of a single neuron, biocytin or Neurobiotin was electroporated from a glass electrode into the recorded neuron with positive current pulses to obtain the morphological information and cortical position of the neuron. The electrode depth was also used to estimate the position of the recorded neurons.

Although the dataset of the previous juxtacellular recordings contains activity of 87 neurons from 69 rats, we only adopted the data recorded from such neurons that yielded sufficiently many $(>500)$ spikes in any of slow $\gamma$, fast $\gamma$, and $\theta$ frequency ranges for the present statistical analysis ( $n=71,61$ rats). This lower bound was chosen to avoid a possible bias in the estimation of the significance of phase locking (the value of $R$ explained later) from the small number of spikes. In the majority of datasets (35 rats), we combined juxtacellular recordings and multiunit recordings with a silicon probe (explained below), which also has 8 single-electrode sites for LFP recordings. In other juxtacellular recordings (26 rats), we conducted LFP recordings with a single electrode placed at the depth of $400-600 \mu \mathrm{m}$ (8 rats) or 1000-1200 $\mu \mathrm{m}$ (18 rats) from the cortical surface. The results of LFP recordings were newly obtained by the present analysis, and the LFP data obtained by an electrode were included in Figures $1 F-I, 4,5,6$, and 7 . The cell types of the neurons that were not successfully identified by juxtacellular recording technique were inferred from the width of recorded spikes (Isomura et al., 2009).

Multiunit recordings. Multiunit recordings were performed on 15 head-restrained behaving rats by using a 16-channel silicon probe with two tetrode sites, one located at the tip of the probe and the other at a distance of $800 \mu \mathrm{m}$ from the tip (see Fig. $1 B$ ), which enabled simultaneous recordings from the superficial layers (typically at the depth of 400 $\mu \mathrm{m}$, corresponding to L2-L3) and the deep layers (typically at the depth of $1200 \mu \mathrm{m}$, corresponding to L5). We also recorded LFP data newly from 12 rats by using the eight electrode sites of the silicon probe that are arranged equidistantly from the tip with a spatial separation of $150 \mu \mathrm{m}$. The tip of the silicon probe was placed at the depth of $\sim 1200 \mu \mathrm{m}$ or $\sim 1600 \mu \mathrm{m}$ from the cortical surface. We used the spike data obtained from the epoch in which the rats were highly active (i.e., within $10 \mathrm{~s}$ before and after every lever pull or lever push). In all the figures showing results of our multiunit recordings, we used the spikes of individual neurons sorted with our in-house spike sorting software based on the robust variational Bayes (Takekawa et al., 2012). Unsorted spikes were not used for any analysis. The software is available at http://etos. sourceforge.net/. We used only such neurons as yielded $>500$ spikes for the analysis of spike phase locking to slow $\gamma$, fast $\gamma$, and $\theta$ oscillations. The LFP recorded at the depth of $450 \mu \mathrm{m}$ was used as the reference for the phase locking. Other details about experimental and analysis methods are found in Isomura et al. (2009).

Functional categorization. The behavior-related spike activity was categorized into five temporally (functionally) different groups according to Isomura et al. (2009). Hold-related activity was a unimodal (increasing, decreasing, or increasing-to-decreasing) activation during the lever holding period. Premovement was a phasic activation starting $<500 \mathrm{~ms}$ before the movement onset and falling down below the half-peak at the movement onset. A movement-related neuron showed a phasic activation during either lever push or lever pull. In general, the neuronal activity of this functional type was strongly selective to the direction of movement. Movement-off was an abrupt drop-off during the movement after constant tonic spiking in the intertrial intervals and lever holding periods. Postmovement was a phasic activation showing a larger peak in the movement-end-aligned histogram than the movement-onsetaligned histogram. The phasic activation/inactivation contained at least three consecutive bins $(20 \mathrm{~ms})$ over $<3$ SDs from the baseline activity $(0.25-1 \mathrm{~s}$ before the movement onset). The categorized activities were further checked by a visual inspection. We occasionally call movementrelated, premovement, and postmovement neurons as " $\mathrm{MOV}^{+}$neurons" because they show similar phasic activation patterns around the time of movement execution. Similarly, hold-related and movement-off neurons, which display sustained firing during lever hold, are termed "HLD ${ }^{+}$neurons."

The above conventional classification of functional neuron subtypes yielded a clear distinction of these subtypes on the basis of the distinct $\theta$ phases of neuronal firing (e.g., Figs. 6C, 7C, and 9B, deep-layer neurons). However, we note that classifying every neuron into a certain functional class requires some caution in view of recent arguments in the monkey motor cortex (Churchland and Shenoy, 2007; Hatsopoulos and Suminski, 2011), where the conventional view of direction-tuned neurons has been challenged because only a minority of them display a simple scaling of firing rate with the velocity of arm movement. In the present study, the neurons that we could not classify into $\mathrm{MOV}^{+}$or $\mathrm{HLD}^{+}$are denoted as "others" (OTH).

Analysis of LFP, current-source density (CSD), and spike phase locking. LFPs were recorded with a bit depth of 16 bits and a sampling rate of 20 $\mathrm{kHz}$, and the row signals were further down-sampled at a rate of $1 \mathrm{kHz}$ through a low-pass filtering $(<500 \mathrm{~Hz})$ by Remez FIR filter. We analyzed the frequency components of LFPs by using the wavelet transform, which is defined as follows:

$$
W(t, s)=s^{-1 / 2} \int_{-\infty}^{+\infty} d t^{\prime} \phi\left(t^{\prime}\right) \psi\left(\frac{t-t^{\prime}}{s}\right)
$$

for a continuous signal $\phi(t)$ typically representing LFP, and Morlet wavelet function $\psi$ is used in the present study (Torrence and Compo, 1998). In the actual calculation, the down-sampled version of $\phi(t)$ was used, and the integration was replaced with a summation over discretized functions. We conducted CSD analysis to find the spatial locations of current sources and sinks along the electrode array. CSD at depth $z$ was defined with the interelectrode distance $h$ as follows:

$$
\frac{d^{2} \phi}{d z^{2}}=\frac{\phi(z+h)-\phi(z-h)-2 \phi(z)}{h^{2}} .
$$

Slow and fast $\gamma$ components were extracted by applying a Remez FIR filter with bandpass frequencies of $30-50 \mathrm{~Hz}$ or $60-120 \mathrm{~Hz}$, respectively, to the down-sampled version of LFPs or CSDs. $\theta$ components were obtained by bandpass filtering for $4-10 \mathrm{~Hz}$. Hilbert transform was used to define the instantaneous amplitude and phase of these oscillations (Thomas, 1969). To detect periods of significantly strong oscillations, we categorized consecutive oscillatory cycles into 10 ranked subgroups according to the oscillation amplitude and regarded oscillatory cycles as significantly oscillatory if their amplitudes are greater than the median. We conducted a Rayleigh test for circular distributions and identify significantly strong phase locking to oscillations if the $z$-value satisfies $p<$ 0.01 (i.e., $z \geq \ln 0.01$ ) (Sirota et al., 2008; Quilichini et al., 2010). If a neuron generated $n$ spikes at phases $\left\{\theta_{i}\right\}_{i=1, \ldots, n^{\prime}}$ we calculated the magnitude of phase locking (i.e., the average vector length) of this neuron as follows:

$$
R=(1 / n) \sum_{i=1}^{n} \theta_{i} .
$$

Because the average vector length obtained in this test can be biased by spike counts and spike train history effects, we examined the significance 
A
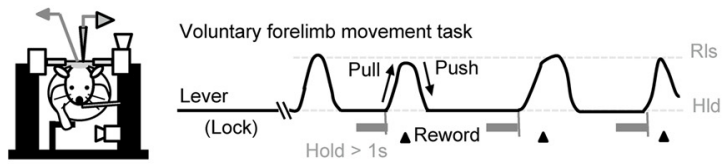

$\mathrm{B}$

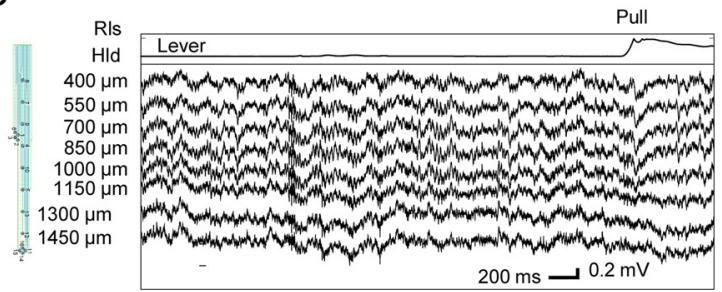

C

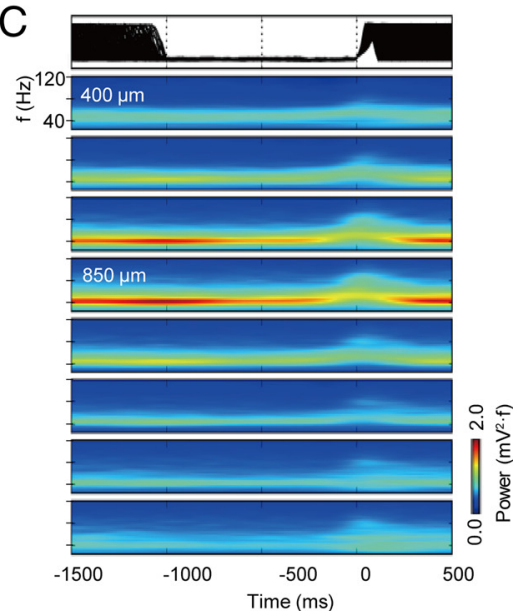

$\mathrm{F}$

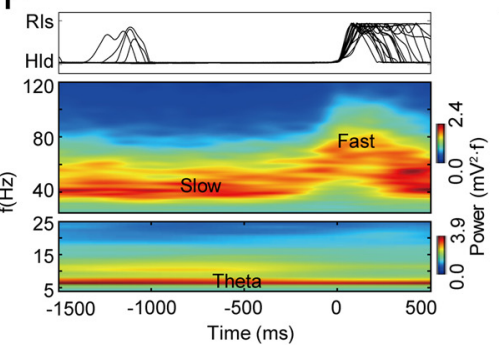

G
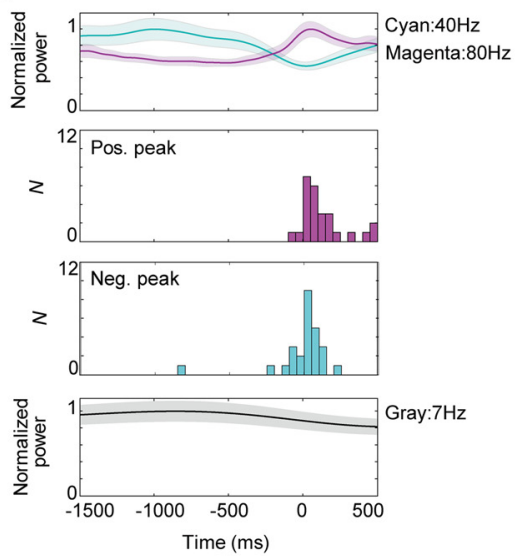

$\mathrm{D}$

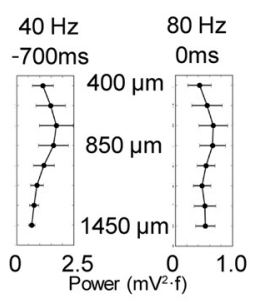

$\mathrm{E}$

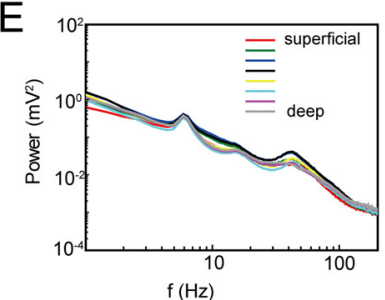

$\mathrm{H}$

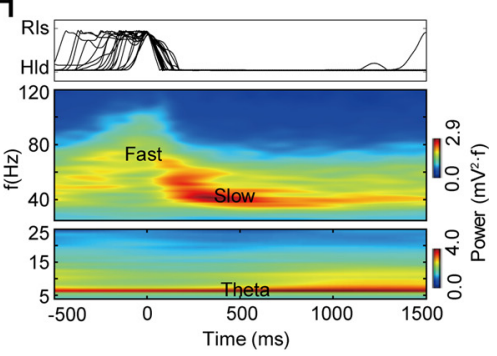

1
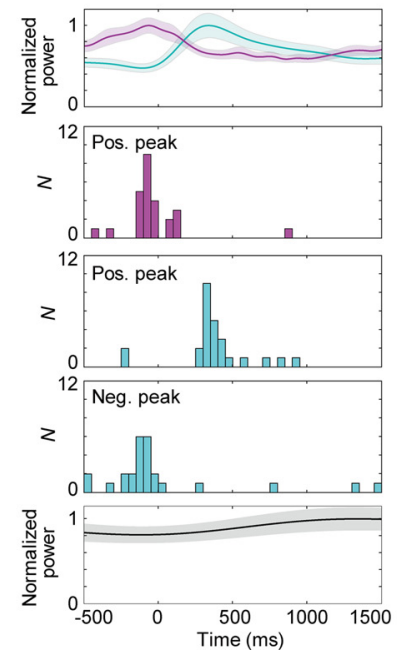

Figure 1. Time-frequency change of LFP $\gamma$ oscillations during sequential voluntary arm movements. $A$, Head-restrained rats performed self-paced sequential lever push/hold/pull movements (left). Rats were rewarded if they pulled the lever after holding of phase locking by using the "pairwise phase consistency" proposed recently (Vinck et al., 2012). Briefly, we randomly sampled 500 to 1500 spikes from each neuron and calculated phase differences between all spike pairs that are temporally separated by $>1 \mathrm{~s}$. Then, we summed up the cosines of these phase differences to obtain pairwise phase consistency, which should be close to unity if spikes are periodic. Both methods yielded almost identical results for both $\gamma$ and $\theta$ oscillations in our dataset. Therefore, below we only show the results obtained by the Rayleigh test.

We consider that the risk of spike contamination is low in the present analysis of spike phase locking to LFPs. In our experiment, spikes were most strongly phase-locked to LFPs during the significantly oscillatory epochs of LFPs, whereas in many neurons spike phase locking occurred also in low-frequency spontaneously firing regimens (e.g., movement-related neurons in slow $\gamma$ regimens or hold-related neurons in fast $\gamma$ regimens). In addition, a juxtacellular pipette and a silicon probe to record LFPs were separated by several hundreds of micrometers to $1 \mathrm{~mm}$ in our juxtacellular recordings.

Principal component analysis. We calculated the PCAs of the CSDs obtained at six different depths as follows. Our analysis of LFPs shows that fast $\gamma$ oscillations appear strongly before and during movement execution, whereas slow $\gamma$ oscillations are strong during the preparatory

\section{$\leftarrow$}

a lever at the push position for $>1 \mathrm{~s}$ (right). $\boldsymbol{B}$, The lever trace (upper) and the raw LFP data (lower) recorded by a silicon probe (left) at various depths of the rat primary motor cortex before and during a pull movement. The probe has two tetrode sites (red circles) and eight electrode sites (arrows). C, The lever positions (top) and the wavelet power spectrums (bottom) calculated at the depths ranging from 400 to $1450 \mu \mathrm{m}$ are aligned with respect to the onsets of repeated pull movements (0 ms). An event-triggered average of WPS was calculated $>345$ trials. $D$, The average maximal powers of 40 and $80 \mathrm{~Hz}$ oscillations are displayed at different depths. The powers were averaged $>12$ rats. The slow and fast $\gamma$ oscillations exhibited maximal powers 700 or $0 \mathrm{~ms}$ before pull movements, respectively. $\boldsymbol{E}$, The power spectrum of the LFP recorded at various cortical depths. $\boldsymbol{F}$, Event-triggered average of the WPS of the LFP recorded by a tetrode at the depth of $600 \mu \mathrm{m}$ (lower) aligned at the onset of pull movements (upper). Top and bottom color bars represent the powers of two frequency bands of $>25 \mathrm{~Hz}$ and $<25 \mathrm{~Hz}$, respectively. They were displayed separately because their power ranges were significantly different. G, The time evolution (mean \pm SE) of the $40 \mathrm{~Hz}$ (cyan) and $80 \mathrm{~Hz}$ (magenta) powers averaged $>26$ rats is displayed around the onset of pull movement (top). Middle two panels, Distributions of the times at which $80 \mathrm{~Hz}$ oscillations exhibit the highest power, or $40 \mathrm{~Hz}$ oscillations exhibit the lowest power. Bottom panel, Time evolution of the $\theta$ power. $\boldsymbol{H}$, Event-triggered average of the WPS at the depth of $600 \mu \mathrm{m}$ (lower) aligned at the onset of push movements (upper).I, The time evolution (mean $\pm \mathrm{SE}$ ) of the power of $40 \mathrm{~Hz}$ (cyan) or 80 $\mathrm{Hz}$ (magenta) oscillations is shown around the onset of pull movement (top). Middle three panels, Distributions of the times at which the $80 \mathrm{~Hz}$ power takes a maximum or the $40 \mathrm{~Hz}$ power takes a maximum or a minimum. Bottom panel, Evolution of the $\theta$ power. 
phase of movements. Therefore, for the fast $\gamma$ oscillations, we used the CSDs obtained in the interval from $200 \mathrm{~ms}$ before to $300 \mathrm{~ms}$ after the onset of each push movement. For the slow $\gamma$ oscillations, we used the data in the interval from 1000 to $500 \mathrm{~ms}$ before the onset of each pull movement. The envelopes of fast and slow $\gamma$ oscillations showed peak amplitudes in the corresponding intervals. Although the amplitude of $\theta$ oscillation undergoes task-related modulations, the change is within $20 \%$ of the maximum. Therefore, the interval for $\theta$ oscillation was taken to be the entire task period. The troughs of oscillations were detected using the CSDs calculated at the depth of $700 \mu \mathrm{m}$. We computed a matrix with each element representing the covariance of the CSDs averaged over the above intervals between all pairs of six electrode sites for each rat (Sukov and Barth, 1998). Eigenvectors of the covariance matrix give the axes in the electrode space that represents the independent sources of covariance, or principal components, between the CSDs. The corresponding eigenvalues give the relative amount of the total variance contributed to by each source. Averaging over animals was obtained by averaging the elements of the covariance matrix across 12 rats.

\section{Results}

Behavior state-dependent switching between slow and fast $\gamma$ oscillations

We analyzed spike data obtained from head-restrained rats that were trained to voluntarily repeat a sequence of push-hold-pull of a lever. Although juxtacellular data and multiunit recording data from the deep layers were reported previously (Isomura et al., 2009), multiunit recording data from the superficial layers and LFP data were newly analyzed here. The rats were rewarded if they successfully held a lever at the hold position for $>1 \mathrm{~s}$ and then pulled it to the release position (Fig. 1A). Rats were not required to keep holding a lever before push movement. LFPs were recorded at different depths of the forelimb area of the rat motor cortex by using either a single electrode or a silicon probe with eight electrodes and two tetrode sites (Fig. $1 B$, left). Spike sequences were juxtacellularly recorded from all layers of the same motor cortical area, and multiunit recordings were simultaneously performed at the superficial and deep layers with the two tetrode sites (for further details, see Materials and Methods).

Figure $1 B$ (right) shows a typical example of the LFPs recorded with a silicon probe at different depths of the rat motor cortex. To identify multiple oscillatory components clearly, we calculated the wavelet power spectrum (WPS) averaged over trials for the LFPs aligned to the onset of pull movement (Fig. 1C). At all depths of the recording, the LFPs exhibited large amplitude oscillations of $\sim 40 \mathrm{~Hz}$ and a faster $\gamma$ component of $\sim 80 \mathrm{~Hz}$. The powers of the two types of $\gamma$ oscillations peak at a depth of $550-$ $850 \mu \mathrm{m}$, which corresponds to L2-L3 and L4 (Isomura et al., 2009, their Supplemental Fig. 7a) in all the 12 animals examined (Fig. 1D). Although the rat motor cortex is generally known to have no distinct L4, the rat forelimb motor area has a L4 (Cho et al., 2004). The power of slow $\gamma$ was generally weak at the depth of $1150-1450 \mu \mathrm{m}$ (in 9 rats), which may correspond to L5B and L6. In Figure $1 E$, we show the power spectrums obtained from the superficial to the deep cortical layers. Whereas the slow $\gamma$ component shows a clear peak, the fast $\gamma$ component only shows a weaker, broader hump presumably because the latter occurs only briefly during the movement execution.

The WPS recorded by a tetrode also reveals that slow $\gamma(30-50$ $\mathrm{Hz}$ ) band is dominant during the lever hold period, whereas fast $\gamma$ band $(60-120 \mathrm{~Hz})$ starts to appear $\sim 100 \mathrm{~ms}$ before the onset of lever pull and ended $\sim 100 \mathrm{~ms}$ after the termination of the movement (Fig. $1 F$ ). In Figure $1 G$, we summarize the exchanges of the slow and fast $\gamma$ powers over the different rats. Similarly, the analysis of the same LFP data aligned to the onset of lever push reveals
A

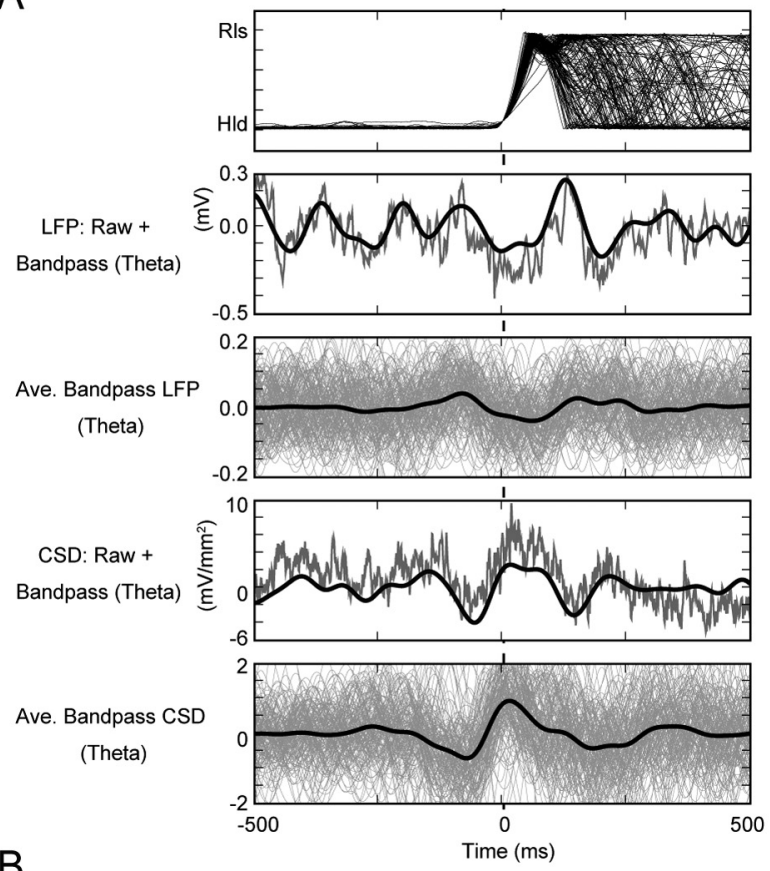

B

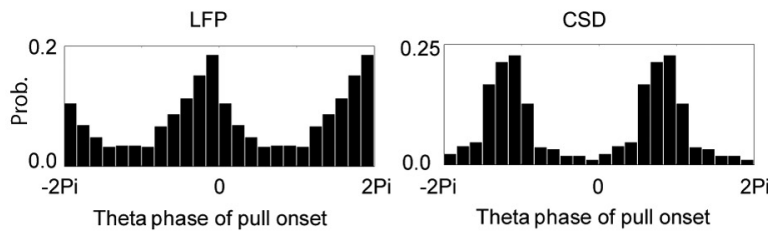

C

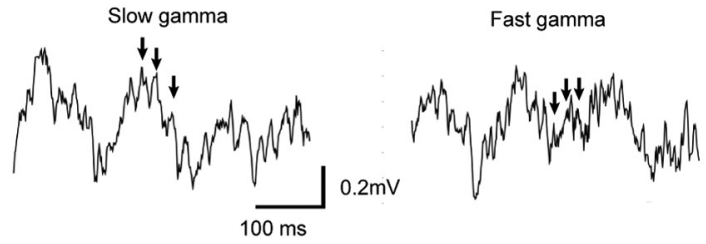

D
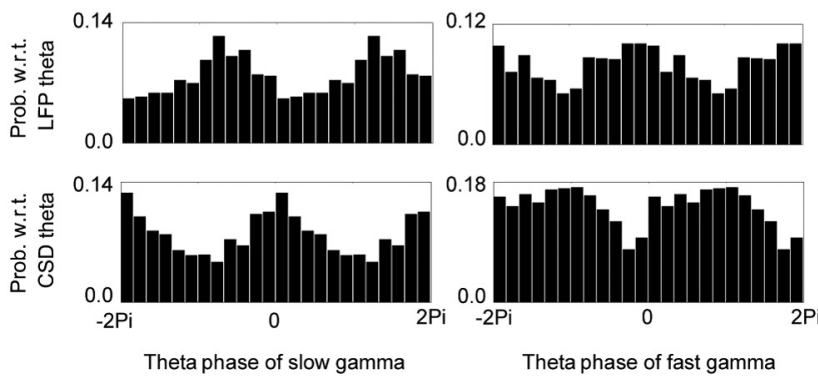

Figure 2. Cross-frequency couplings between $\theta$ and $\gamma$ LFP oscillations. A, Top, Lever trace in repeated trials. Second panel, Raw (gray) and bandpass-filtered (black) traces of the LFP in a trial. Third panel, Bandpass-filtered LFP traces in repeated trials (gray) and their average (black). Fourth and fifth panels, Similar traces of the CSD in a single trial and repeated trials, respectively. The band frequencies are $4-10 \mathrm{~Hz}$ for $\theta$ oscillation and $30-50 \mathrm{~Hz}$ and $60-120 \mathrm{~Hz}$ for slow and fast $\gamma$ oscillations, respectively, and all these trials were performed by the same rat. The LFP signals were recorded at the depth of $700 \mu \mathrm{m}$ and are aligned to the onset of lever pull. $B$, The distributions of the times of movement onset are shown for lever pull with respect to LFP and CSD oscillations. The movement onsets are significantly time-locked to the troughs of LFP $\theta$ oscillation. C, The LFPs recorded during strong slow or fast $\gamma$ oscillation nested in $\theta$ oscillations are shown. Arrows indicate peaks of a few $\gamma$ cycles. $\boldsymbol{D}$, The distributions of the $\theta$ phases at which the envelope of slow or fast $\gamma$ band exhibits a maximal amplitude: the $\gamma$ band shows a peak power, shown with respect to LFP (upper) and CSD (lower). 
A

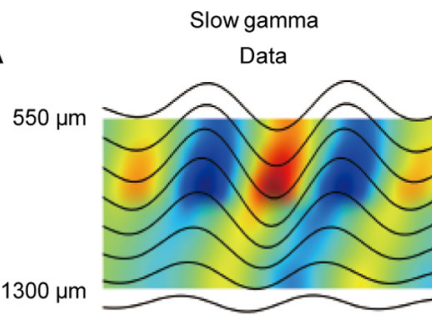

B

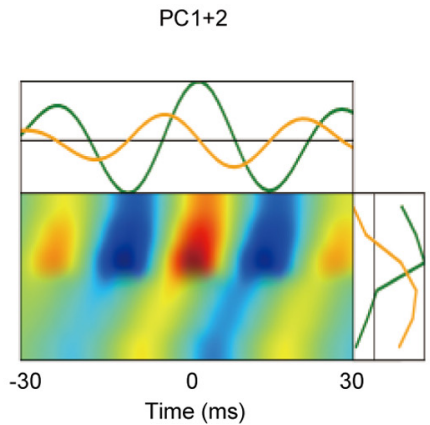

C

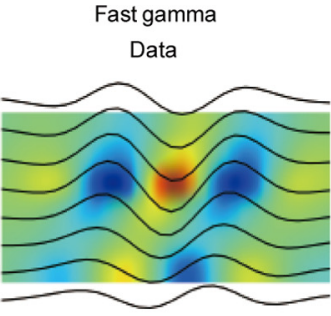

D

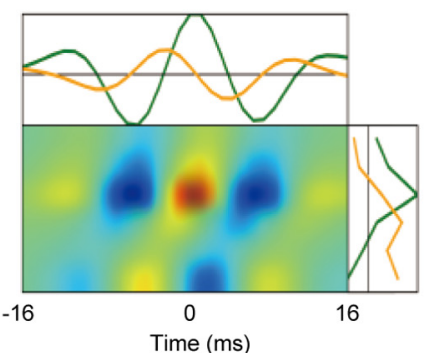

E

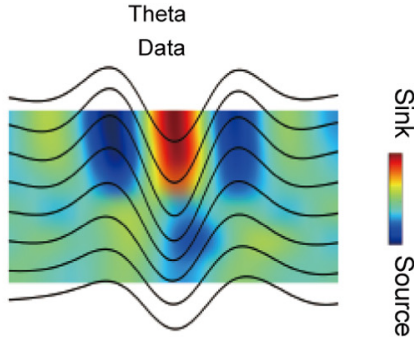

F

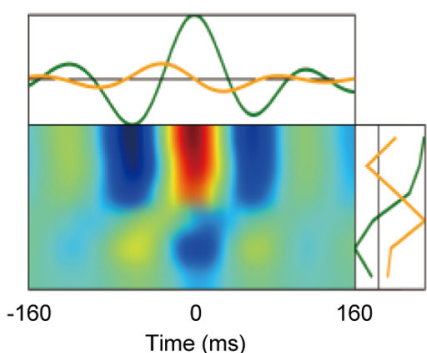

Figure 3. Principal components of the laminar CSD. The LFP data recorded from intervals of 1000 to 500 ms before each lever pull were used for slow $\gamma$ oscillations, whereas those recorded from -200 to $300 \mathrm{~ms}$ after each lever pull were used for fast $\gamma$ oscillations. The data from the entire task period were used for $\theta$ oscillation. $\boldsymbol{A}$, The raw (SD (pseudo-color) and oscillations in LFPs (traces) are shown for slow $\gamma$. $\boldsymbol{B}$, The first (green) and second PCs (orange) are presented (top) together with the corresponding eigenvectors (right). The eigenvectors show the depths at which the PCs are strong. Pseudo-color representation displays the sum of the products of the PCs and the corresponding eigenvectors. The sum was averaged $>12$ rats. $\boldsymbol{C}-\boldsymbol{F}$, Similar diagrams are shown for fast $\gamma$ and $\theta$ oscillations. The sum of the averaged PC1 and PC2 accounted for $>95 \%$ of the variance of CSDs in $\boldsymbol{B}$ and $\boldsymbol{D}$, whereas PC1 accounted for $>95 \%$ of the variances in $\boldsymbol{F}$.

that the power of fast $\gamma$ broadly peaks around the time of lever push (Fig. $1 H)$. The power of slow $\gamma$, which once decreases toward the onset of lever push, tends to exhibit a broad peak $\sim 400$ ms after lever push (Fig. 1I), possibly indicating the beginning of preparation for lever pull or the anticipation of reward.

We analyzed the LFP recorded during no-hold periods. We defined that a rat was in no-hold periods if the rat did not hold a lever for $>1 \mathrm{~s}$ and repeated such behavior $>50$ times. In a nohold period, the lever returns automatically to the pull position (base position). Six rats among 26 displayed no-hold periods, and neural data displayed some extent of individual animal differences. However, some datasets showed evidence for an increased power in slow $\gamma$ band during no-hold periods as well as in lever hold periods (data not shown), suggesting that slow $\gamma$ is associated with the resting behavior of the rats. However, forelimb movements were not quantitatively monitored during nonhold periods, and the relationship between slow $\gamma$ oscillation and the resting behavior of rats remains to be further clarified.

\section{Cross-frequency coupling between $\boldsymbol{\gamma}$ and $\boldsymbol{\theta}$ oscillations}

We noticed that the power spectrum shown in Figure $1 E$ also exhibits a significant peak in the $\theta$ band $(4-10 \mathrm{~Hz}$ : a peak at $\sim 7$ $\mathrm{Hz}$ ). Actually, significant $\theta$-oscillation components were seen at all the cortical depths in all the 12 rats recorded. In Figure $1 F, H$, we show the $\theta$ powers in the LFP data aligned to the onset of lever pull and push, respectively, for a rat. Similar results are more clearly seen in Figure $1 G, I$ (bottom) after averaging over different rats. As seen from these figures, $\theta$ oscillation was prominent during the lever hold period, whereas its amplitude was reduced by $\sim 20 \%$ during the execution of movement. However, these components may represent the influence of volume conduction from distant brain areas. To eliminate such a possibility, we performed CSD analysis at various cortical depths (Materials and Methods).

Figure $2 A$ presents typical examples of the raw and $\theta$ bandfiltered traces of CSDs together with LFPs (see also Fig. $3 E$ ) and the lever trace from a rat. The bandpass-filtered LFP and CSD exhibit a clear tendency of oscillations at $\sim 7 \mathrm{~Hz}$ during the entire period of sequential motor behavior. Then, we calculated the distribution of the phases of pull onset over trials and found that the initiation of lever pull tends to occur at a particular phase of LFP $\theta$ oscillation in this rat (Fig. $2 B$; see also Fig. $9 C, D$ ), as is also seen from the enhanced amplitude of the averaged CSD around the pull onset (Fig. 2A, $0 \mathrm{~ms}$ ). Actually, pull onset was strongly time-locked to certain phases of CSD $\theta$ in all 12 rats $(p<0.01$, Rayleigh test for the circular distributions of pull timing) with the mean magnitude of phase locking $r=0.37 \pm 0.18$ (see Materials and Methods). Moreover, pull onset was phase-locked to LFP $\theta$ in 11 rats, with the preferable phases of pull onset approximately coincided with a trough of LFP $\theta(18 \pm 53$ degrees, $r=0.29 \pm$ 0.09 ). The initiation of lever push also exhibited similar phase locking (CSD $\theta, r=0.42 \pm 0.17,>10$ rats; LFP trough, $16 \pm 36$ degrees, $r=0.33 \pm 0.12$, $>9$ rats). To our surprise, $\theta$ oscillation in motor cortex nests fast and slow $\gamma$ oscillations in a manner similar to hippocampal $\theta$ and $\gamma$ oscillations (Fig. $2 C$ ). To see this, we calculated the amplitude envelopes of $\gamma$ oscillations and constructed histograms of the $\theta$ phases at which the envelopes are maximized in a $\theta$ cycle. The maximum amplitudes of slow $(r=$ $0.14 \pm 0.04,10$ rats $)$ and fast $(r=0.11 \pm 0.02,7$ rats $) \gamma$ oscillations tend to be phase-locked to the $\theta$ oscillation of CSD $(p<$ 0.01 , Rayleigh test) with relative phase differences of $91 \pm 35$ degrees (Fig. 2D). These values are slightly larger than those observed in hippocampus (Colgin et al., 2009). Our results indicate an essential relevance of $\theta$ oscillation to the temporal coordination of voluntary movement.

Because LFP is considered to reflect local synaptic inputs, its laminar pattern contains crucial information on synaptic input to each cortical layer. Figure $3 A, C$ shows CSDs at different depths averaged over slow and fast $\gamma$ cycles, respectively, together with the band-passed LFPs from which the CSDs were calculated. Both $\gamma$ band oscillations exhibit a monotonic phase delay from the 
A
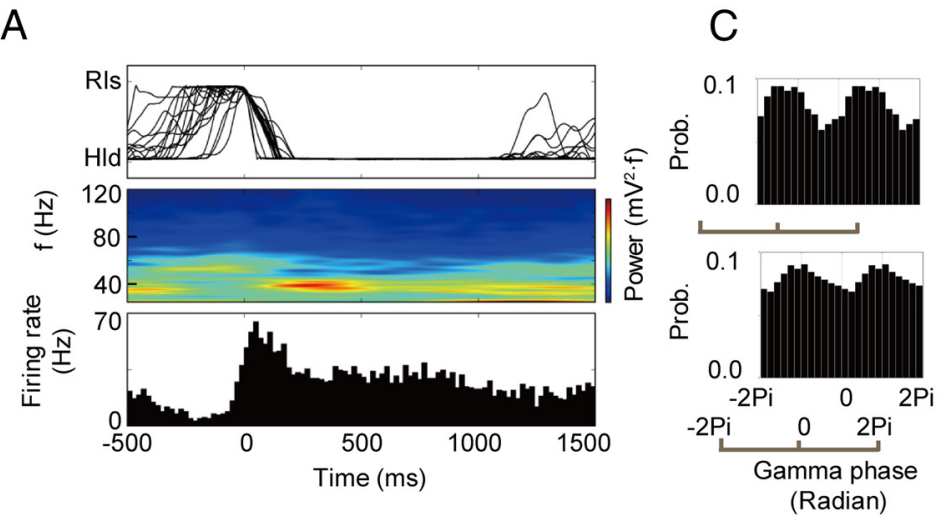

B Rls
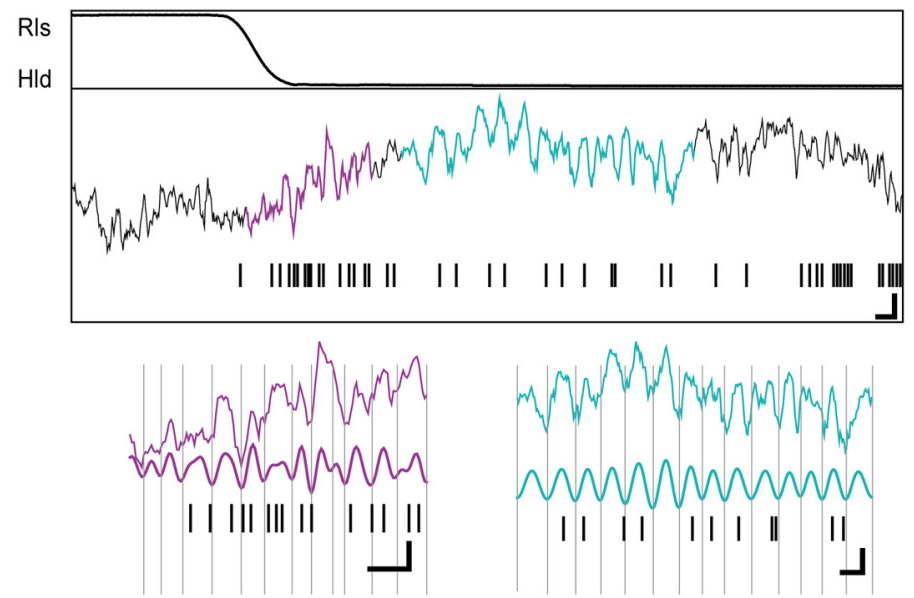

$\mathrm{D}$

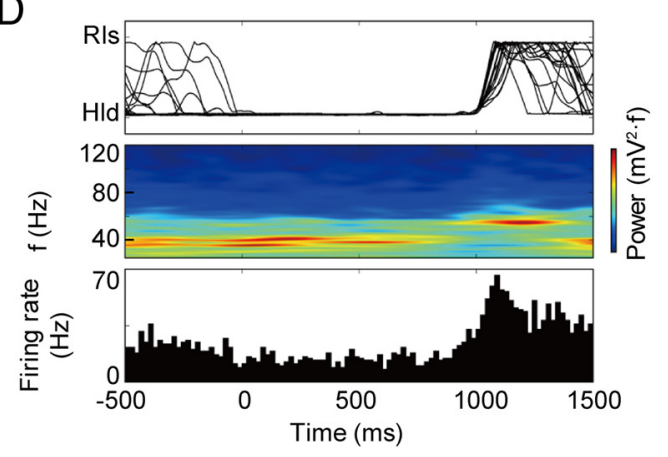

$\mathrm{F}$
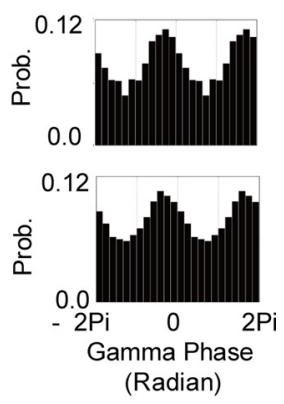

E

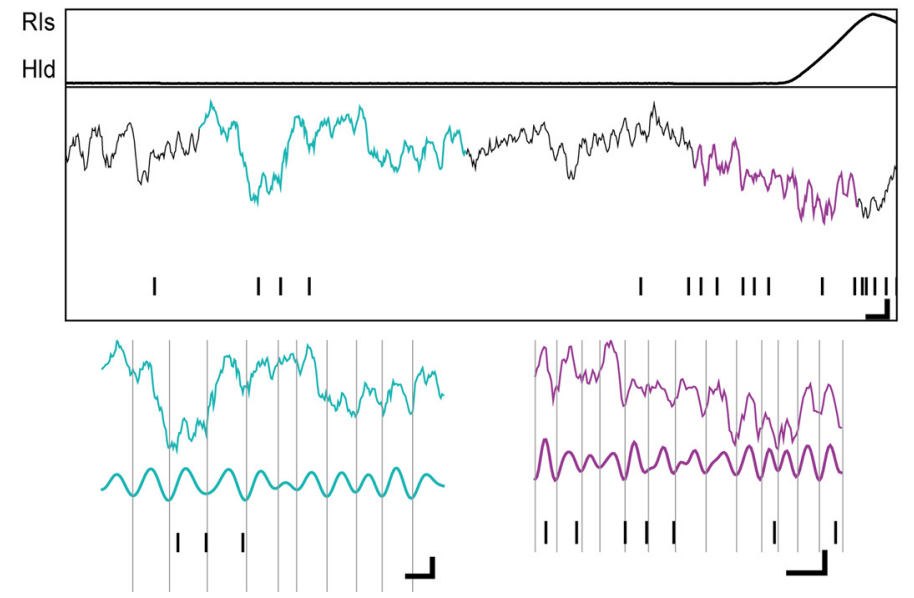

Figure 4. Phase locking of motor cortex neurons to $\gamma$ oscillation of LFP. LFPs were recorded at the depth of $600 \mu \mathrm{m}$. LFPs, calibration: $20 \mathrm{~ms}, 0.5 \mathrm{mV}$. $A$, The lever position (upper), event-triggered average of the WPS of LFP (middle), and PETH (bottom) of an $\mathrm{L} 6$ movement-related pyramidal cell $(1539 \mu \mathrm{m})$ of motor cortex. The PETH shows that this neuron is active during movement execution. The data were aligned to the onset of push movements in different trials. $\boldsymbol{B}$, Spikes of the neuron are shown during the deep to the superficial cortical layers without a clear point of polarity reversal. To explore the underlying mechanism, we conducted principal component analysis (PCA) of the laminar CSDs (Materials and Methods). We found in both $\gamma$ bands that $\mathrm{PC} 1$ predominantly appears in the superficial layer and PC2 is dominant in the deep layers, and that PC2 is more advanced than PC1 by $\sim 90$ degrees (Fig. $3 B$ vs Fig. 3D). Thus, two independent sinksource pairs of the membrane current, one in the superficial layer and one in the deeper layer, underlie the laminar phase shifts of the two bands. Similar results are known for slow $\gamma$ oscillations in the auditory cortex of anesthetized rats (Sukov and Barth, 1998). In contrast to $\gamma$ oscillations, the phase of $\theta$ oscillation did not depend significantly on the depth and a single sink-source pair dominating at the superficial layer was sufficient to account for the laminar pattern (Fig. $3 E, F$ ).

\section{$\gamma$ phase-locked spiking of motor cortical neurons}

We now show that fast and slow $\gamma$ oscillations of the LFP entrain the spike firing of juxtacellularly recorded excitatory and inhibitory neurons in motor cortex. During the juxtacellular recordings, we simultaneously recorded LFPs primarily at the depths of $\sim 500 \mu \mathrm{m}$ or $\sim 1 \mathrm{~mm}$. As shown above, the phase of $\gamma$ oscillations shifts with the depth. Therefore, in some results, we converted the $\gamma$ phases to the values expected at the depth of cell bodies of the juxtacellularly recorded neurons by using the average phase differences obtained in Figure 3.

We categorized each cell type into five functional neuronal subtypes: movementrelated, premovement, postmovement, hold-related, and movement-off neurons

\section{$\leftarrow$}

epochs of distinct slow (cyan) and fast (magenta) $\gamma$ oscillations together with the lever trace. The significantly oscillatory epochs are magnified and presented with filtered traces to show spike phase locking clearly, where vertical gray lines indicate the phase zero of each $\gamma$ cycle. $\boldsymbol{C}$, The phase distributions of spike firing demonstrate that the neuron has preferable phases of firing in slow (upper) and fast (lower) $\gamma$ bands. Grayscales designate the oscillation phases at the recording site and black ones those converted to L6. D, The lever position (top), event-triggered average of the WPS of LFP (middle), and the PETH (bottom) of an FS interneuron in the layer 3 (depth $658 \mu \mathrm{m}$ ). The data are aligned to the onset of the lever pull, for which this neuron was strongly activated. $\boldsymbol{E}$, Spikes of the neuron are shown during the epochs of distinct slow (cyan) and fast (magenta) $\gamma$ oscillations together with the lever trace. Scale bars and the depth of recordings are the same as in $\boldsymbol{B}$. $\boldsymbol{F}$, The distributions of firing phases of this neuron in successive slow (upper) or fast (lower) $\gamma$ cycles. 
A

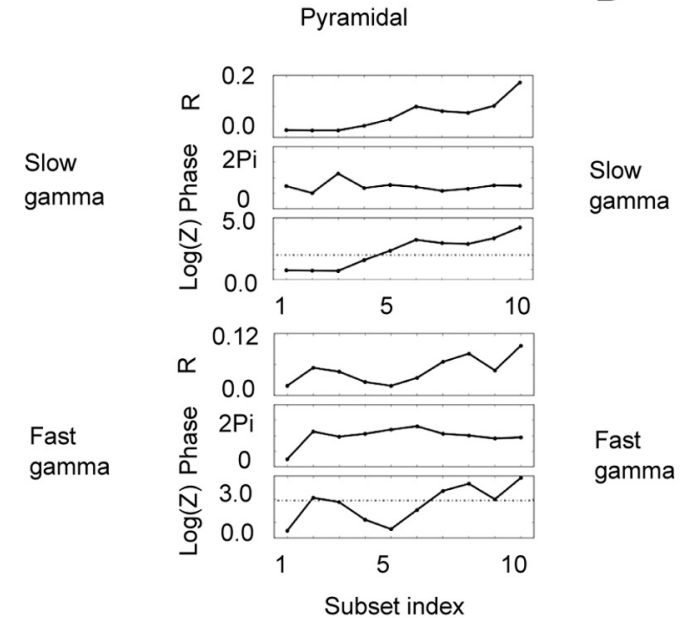

FS interneuron
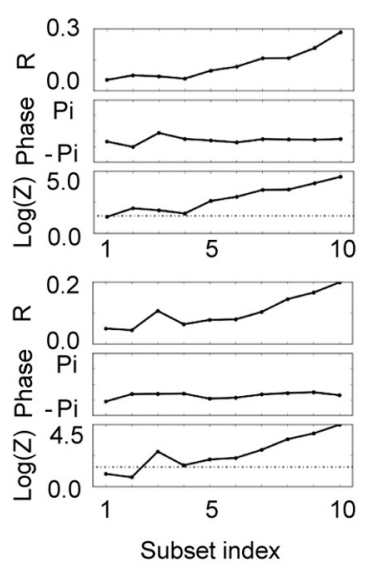

C-1

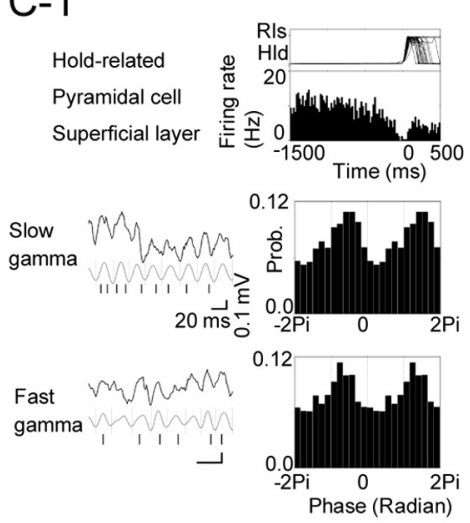

C-2

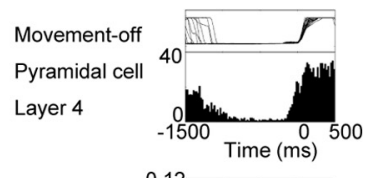

C-3

Movement-related
Pyramidal cell
Layer 5A

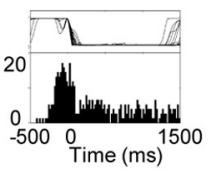

C-4

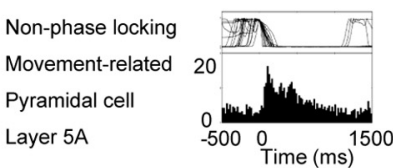

Layer $5 \mathrm{~A}$
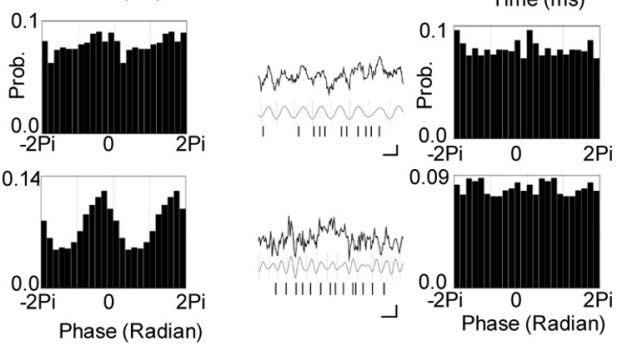

C-5

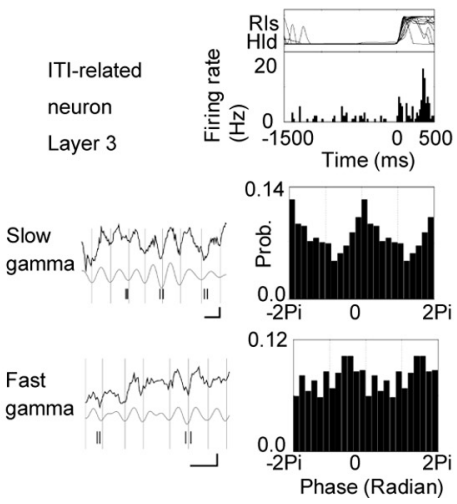

C-6
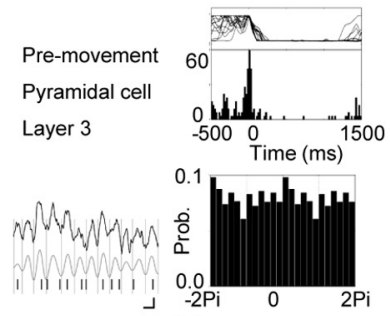

C-7

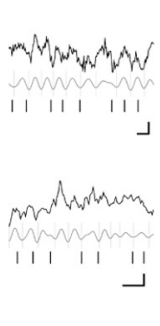

C-8

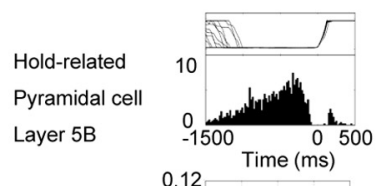

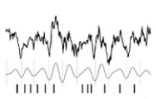
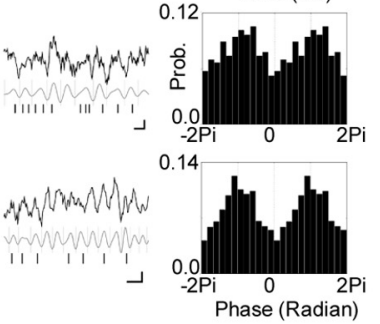

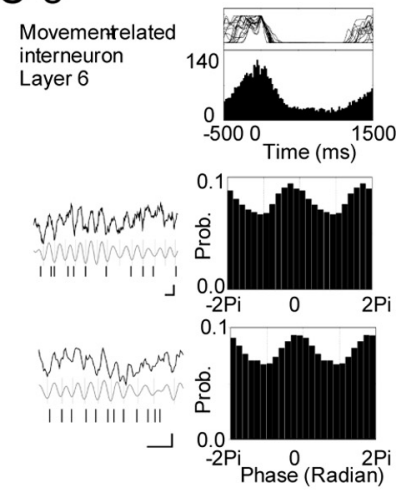

Figure 5. $\quad \gamma$ phase locking across cell types and layers. $\boldsymbol{A}$, The relationships between the magnitude of spike phase locking to LFP oscillations (top), spiking phases of a juxtacellulary recorded pyramidal neuron (middle), and the $z$-score of Rayleigh test (bottom) averaged over repeated $\gamma$ cycles. The upper and lower sets of three panels are for the slow and fast $\gamma$ oscillations, respectively. The LFP was first filtered for either slow or fast $\gamma$ (Materials and Methods); then the LFP amplitudes were divided into 10 equal-sized subsets for each frequency band. The greater the subset index, the stronger the oscillatory tendency. Dotted lines show $1 \%$ confidence level $(p<0.01)$, and only the intervals of LFPs with indices $>5$ were included in the analysis of phase-locked spikes. $\boldsymbol{B}$, Similar quantities were calculated for a juxtacellulary recorded FS interneuron. (-1-C-8, Phase-locked firing is shown for various task-related pyramidal cells and FS interneurons. In all figures, right panels represent the lever trace (top upper), firing activity aligned at the onset of either lever pull or lever push (top lower), and the distributions of spiking phases in slow (middle) and fast $\gamma$ bands (bottom). Left panels represent LFPs, bandpass-filtered $\gamma$ oscillations, and spike times.

(Materials and Methods). Figure $4 A$ displays the perievent time spike histogram (PETH) of a juxtacellularly recorded L6 movementrelated pyramidal neuron together with arm trajectories and the WPS of LFPs. The PETH is the histogram of trial-by-trial spike trains aligned at the time of a behavioral event (here, the onset of lever push). As shown previously, the two $\gamma$ bands exchange around the time of push movement. Figure $4 B$ displays a spike train of the py- ramidal neuron and the time evolution of LFP in a single trial. During significantly oscillatory periods, most spikes were generated at approximately fixed phases of fast (during movement execution) and slow (during lever holding) $\gamma$ cycles. Spikes occurred preferentially near the troughs of both slow and fast $\gamma$ oscillations if their phase was converted to value expected at the depth corresponding to L6 (Fig. 4C). A similar example of phase-locked spiking is shown for 
A
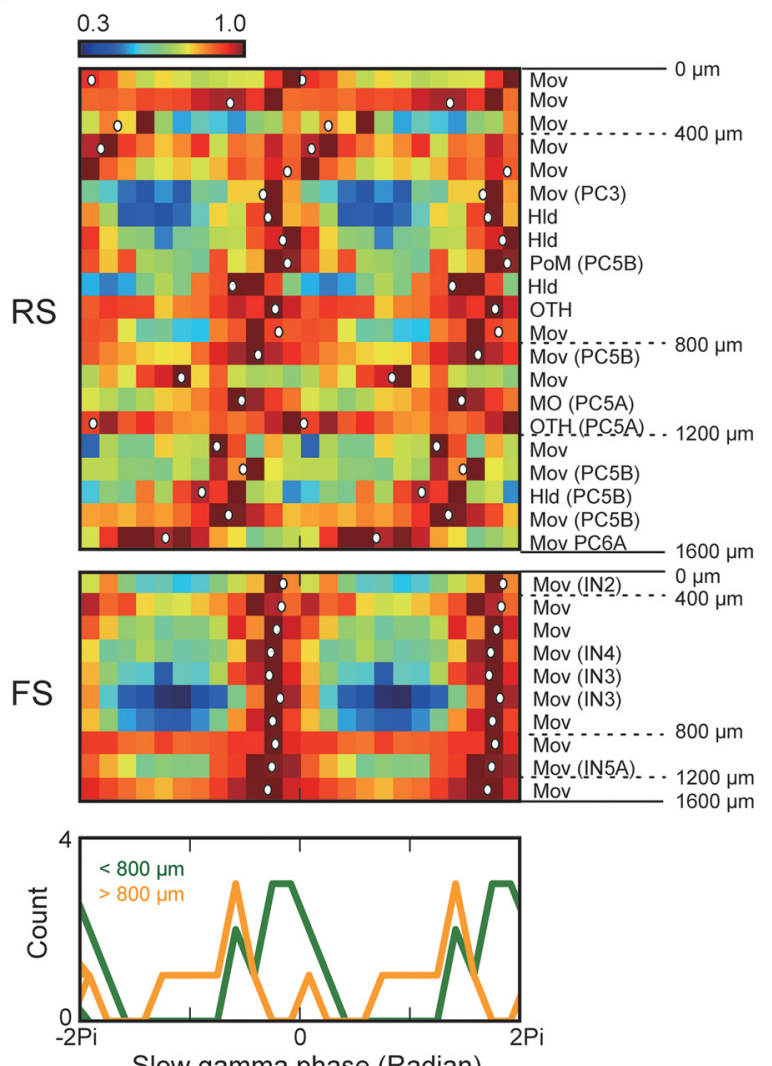

Slow gamma phase (Radian)

C
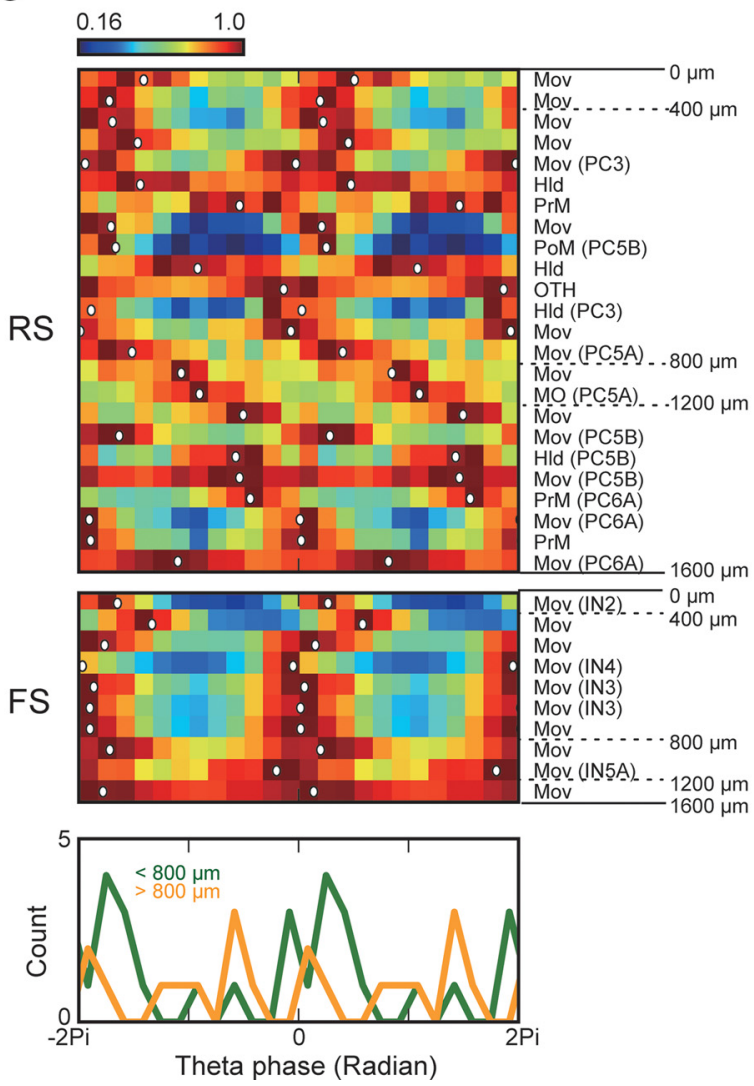

B
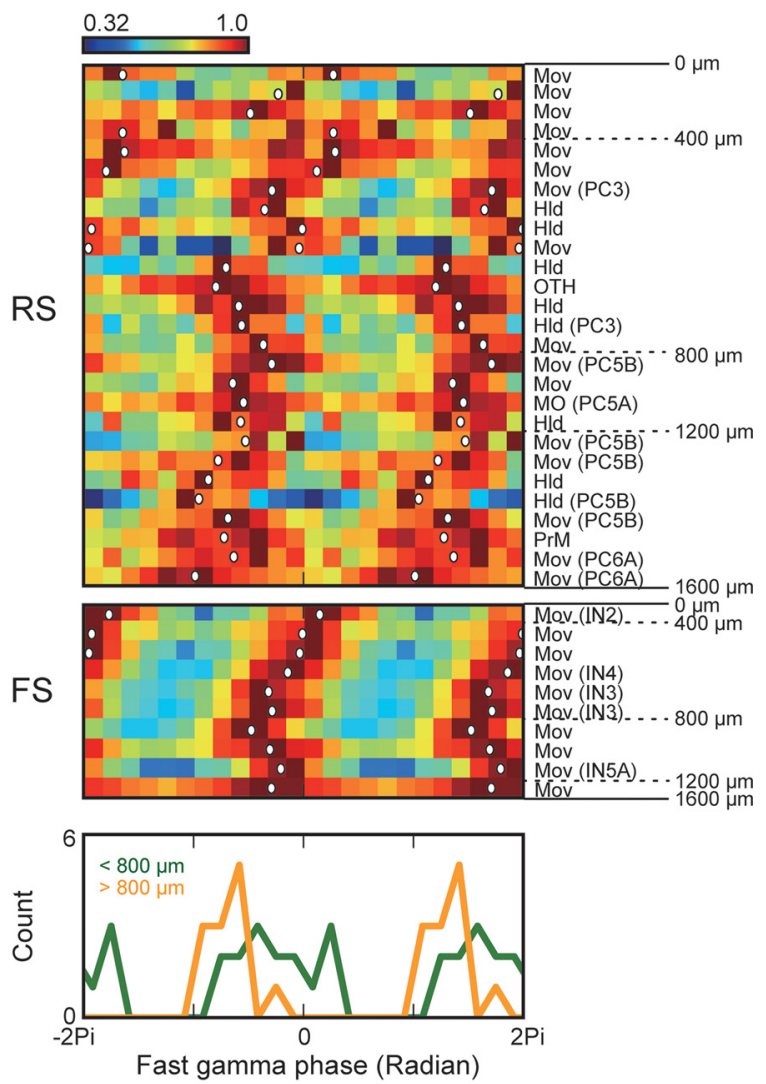
A

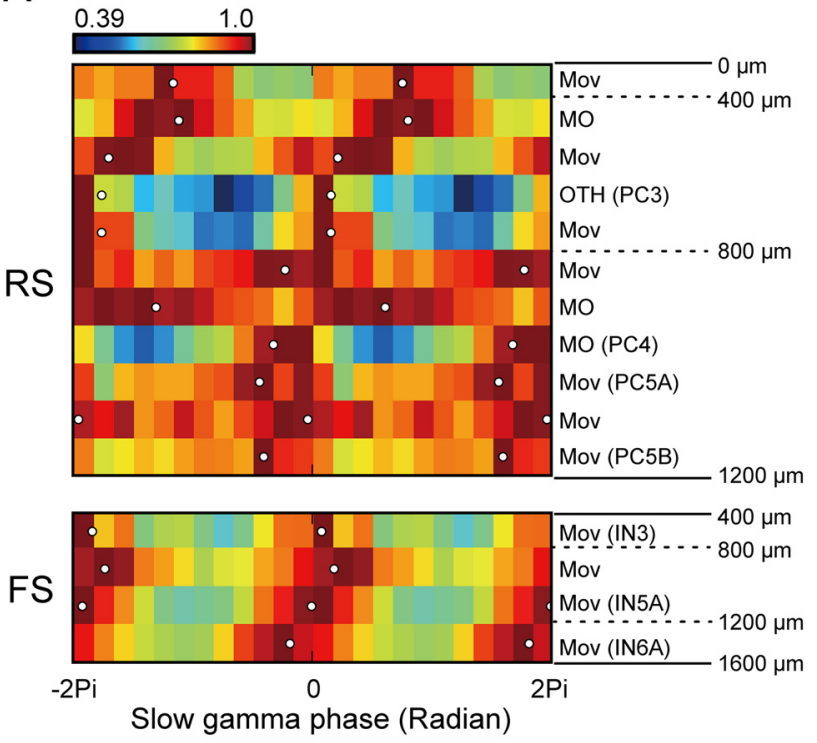

C

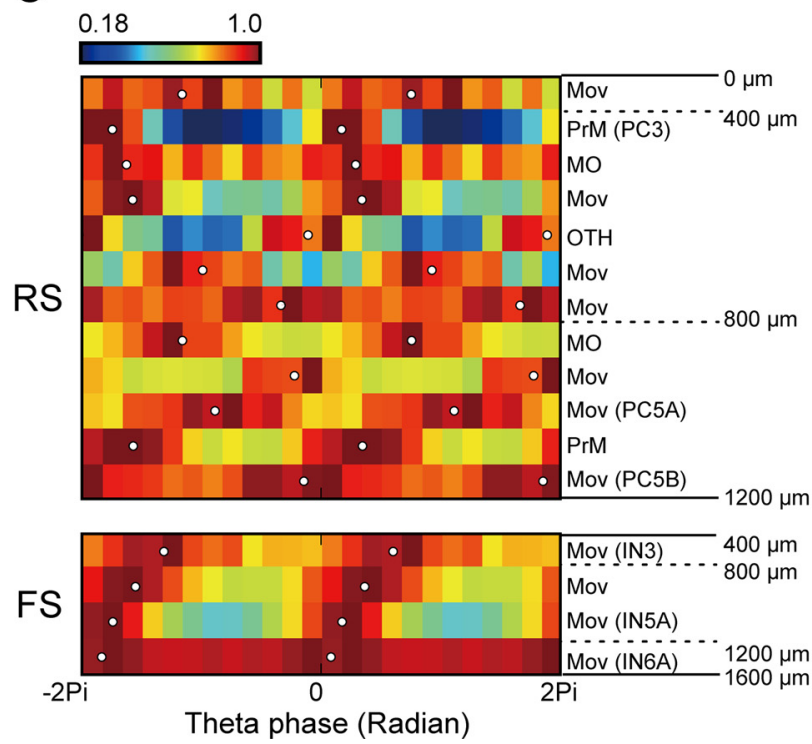

B

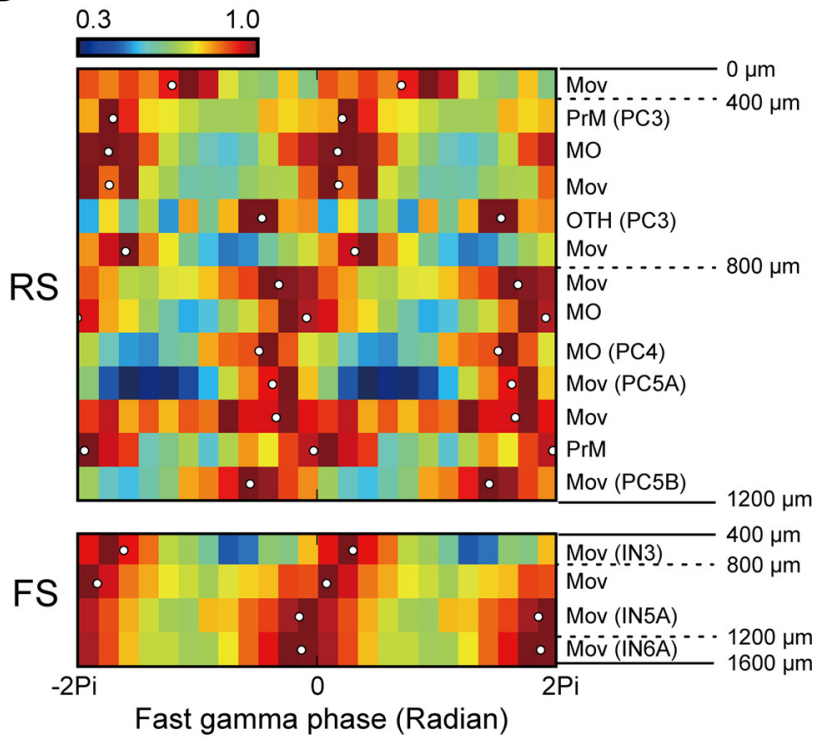

Figure 7. Juxtacellularly recorded neurons phase-locking to LFP at the deep layer. $\boldsymbol{A}-\boldsymbol{C}$, The spike density distributions of individual neurons are shown with respect to the oscillation phases of slow $\gamma$, fast $\gamma$, and $\theta$ oscillations, respectively. The LFPs were recorded at the cortical depth of $1150 \mu \mathrm{m}$, and the depth of the cell body is shown for the individual neurons. The cell type of each neuron is shown when its morphology was successfully identified, or otherwise neurons were classified into RS (tops) and FS (bottom) according to their spike widths.

a FS interneuron in L3 (Fig. $4 D, E$ ), which also preferentially fired at the troughs of $\gamma$ oscillations (Fig. $4 F$ ). This interneuron type is most frequently activated during movement execution. In general, FS interneurons showed a larger maximum probability of phase locking and smaller fluctuations in the spiking phase than pyramidal neurons (compare Fig. 4C,F). Although pyramidal neurons skip more $\gamma$ cycles without firing than FS interneurons, the ratio of spikes within strongly oscillatory epochs to all spikes in the entire task period was

$\leftarrow$

(Figure legend continued.) identified, or otherwise neurons were classified into RS (tops) and FS (bottom) according to their spike widths. For each cell type, neurons were aligned according to the depths of their cell bodies. White dots indicate the average phases of peak firing probability (top, middle), and the distributions of the average peak phases are shown for RS cells in the superficial (green represents depth $<800 \mu \mathrm{m}$ ) and deep (orange represents depth $>800$ $\mu \mathrm{m})$ layers (bottom) not significantly different between pyramidal (47.1 $\pm 5.7 \%$ for slow; $54.0 \pm 4.4 \%$ for fast $\gamma$ ) and FS neurons ( $44.7 \pm 3.5 \%$ for slow; $55.5 \pm$ $3.3 \%$ for fast $\gamma$ ).

In both pyramidal neurons and FS interneurons, the tendency of phase-locked firing was stronger when the oscillation amplitude was larger (Fig. 5A,B). The majority of motor cortical neurons exhibited similar phase-locking phenomena regardless of their morphological features and functional subtypes (Fig. 5C). The depth of a cell body also did not significantly affect the tendency of phase locking. Among the juxtacellularly recorded 87 neurons, we analyzed 71 neurons (pyramidal 56, FS 15) that produced sufficiently many spikes $(>500)$ during the significantly oscillatory epochs. Then, 46 neurons (65\%) showed significant phase locking to slow $\gamma$ oscillations, 54 neurons (76\%) to fast $\gamma$, and 43 neurons (61\%), including 14 FS interneurons, to both $\gamma$ bands. These 29 pyramidal neurons $(43-14)$ contain 
A

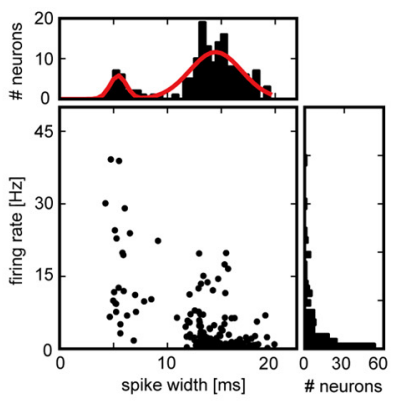

C

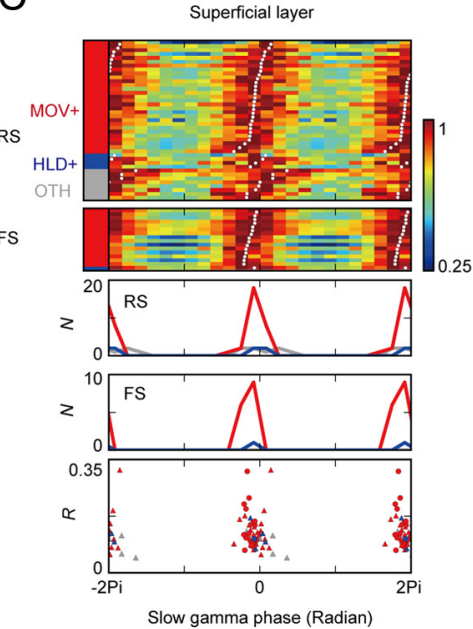

D
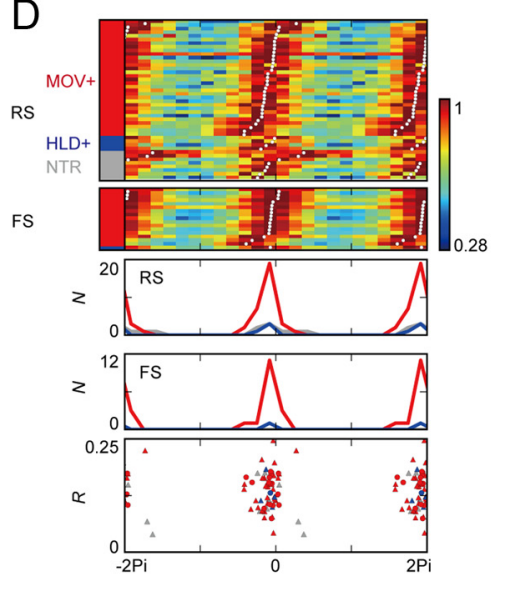

G

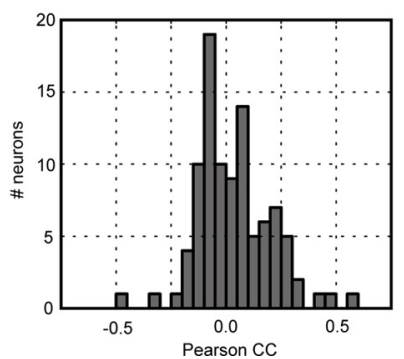

B

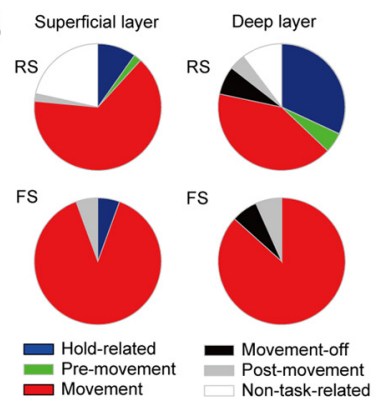

E

RS

FS
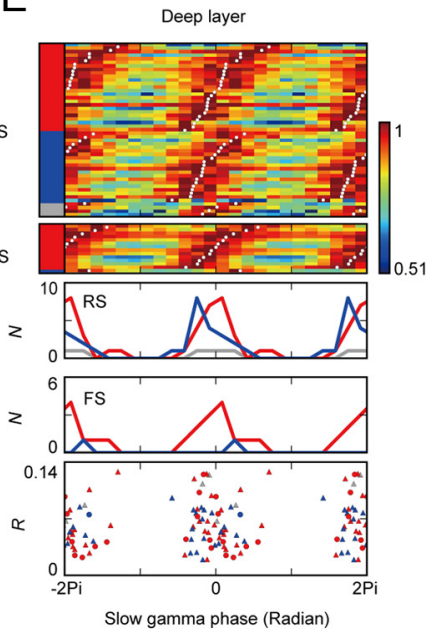

F

RS

FS
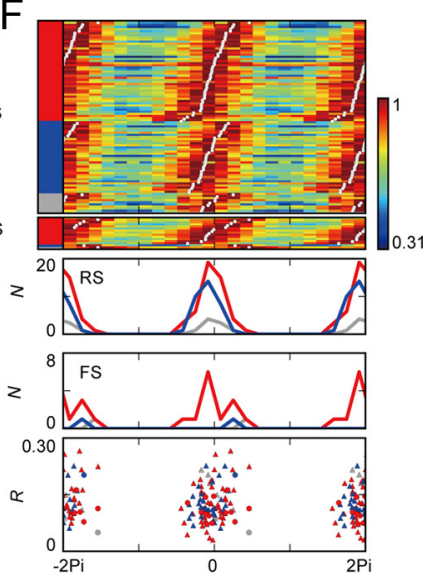

Fast gamma phase (Radian)

$\mathrm{H}$

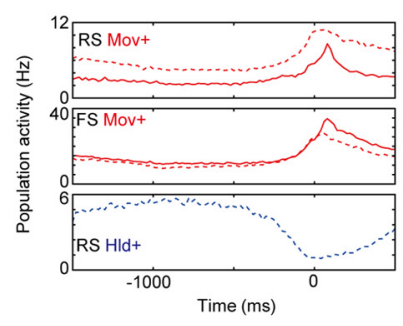

Figure 8. Phase-locking of simultaneously recorded neurons in slow and fast $\gamma$ oscillations. $\boldsymbol{A}$, The distribution of firing rates and spike widths is shown over the neurons recorded with the tetrode sites of a silicon probe. Putative pyramidal neurons show broad spike widths, whereas putative FS interneurons exhibit narrower spike widths. The boundary between the two neuron classes was determined to minimize the classification error, which is defined as the probability that the classification is wrong for all neurons. $\boldsymbol{B}$, The portions of the five functional neuronal subtypes are shown for RS neurons and putative FS interneurons in the superficial and deep layers. $\boldsymbol{C}, \boldsymbol{D}$, Density functions of spiking phase relative to slow and fast $\gamma$ oscillations, respectively, are shown for $\mathrm{MOV}^{+}$(red), $\mathrm{HLD}^{+}$(blue), and other (OTH; gray) RS neurons and FS interneurons in the superficial layer. LFP oscillations measured at the depth of $450 \mu \mathrm{m}$ were used for determining the spiking phase. Activity of a single neuron is displayed in each row

movement-related ( $n=19)$, hold-related $(n=4)$, movement-off $(n=4)$ and nontask-related $(n=2)$ neurons, including an intertrial-interval-related neuron.

Figure $6 A, B$ shows the distributions of spiking phases with respect to slow and fast $\gamma$ oscillations, respectively, for the juxtacellularly recorded neurons that exhibited significant phase locking $(p<$ 0.01 ). Approximately half of these neurons were morphologically identified; and as previously reported, all functional neuronal subtypes appeared at different depths. Pyramidal and FS neurons fired near the troughs of fast and slow $\gamma$ oscillations in the upper layers, and the spiking phase was gradually advanced with the depth because the phase of LFP oscillation was also advanced. In contrast, $\theta$ phaselocked spiking of pyramidal neurons $(n=$ 36) shows an interesting layer dependence (Fig. 6C). Pyramidal neurons in the superficial layers (depths $<800 \mu \mathrm{m}$ ) and FS interneurons in all layers $(n=14)$ fired near the troughs of $\theta$ oscillation. In contrast, spikes were distributed broadly over a $\theta$ cycle in the pyramidal neurons obtained from the deep layers (depths $>800$ $\mu \mathrm{m})$. To see these differences quantitatively, we calculated the distributions of spiking phases separately for neurons in the superficial and deep cortical layers (Fig. $6 A-C$, bottom). The distributions will be later compared with those calculated from multiunit recording data. Similar results of phase-locked firing of juxtacellularly recorded neurons are shown for a reference LFP measured at a different depth (Fig. 7).

\section{$\leftarrow$}

and an empty circle represents the mean firing phase of each neuron, and neurons are arranged in the average temporal order of firing in each functional neuronal subtype. In the superficial layer, 41 of $46 \mathrm{RS}$ and 16 of $17 \mathrm{FS}$ neurons exhibited significant phase locking to slow $\gamma$ and 44 of 46 RS and 17 of 17 FS neurons to fast $\gamma$. The instantaneous number of active neurons is shown for the three functional subtypes of RS neurons (upper middle) and putative FS neurons (lower middle). The coloring of curves indicates the corresponding functional subtype. The degrees of phase-locked firing are also shown for the individual neurons (bottom: $R S$, triangles; putative $F S$, circles). $\boldsymbol{E}, \boldsymbol{F}$, Similar quantities are shown for slow and fast $\gamma$ spiking phases, respectively, in deep layer neurons. LFP oscillations recorded at the depth of $1200 \mu \mathrm{m}$ were used as a reference for the spiking phase. In the deep layer, 49 of $95 \mathrm{RS}$ and 14 of $15 \mathrm{FS}$ neurons exhibited significant phase locking to slow $\gamma$ and 91 of $95 \mathrm{RS}$ and 15 of $15 \mathrm{FS}$ neurons to fast $\gamma$. G, Distribution of correlations between firing rate and the duration of lever push over the neurons recorded with tetrode sites. $\boldsymbol{H}$, The average firing rates are shown for $\mathrm{MOV}^{+} \mathrm{RS}$ neurons (top), putative FS interneurons (middle), and HLD ${ }^{+}$RS neurons (bottom) in the superficial (solid) and deep layers (dashed). The activities are displayed around the onset of lever pull (time 0 ). 
A Superficial layer
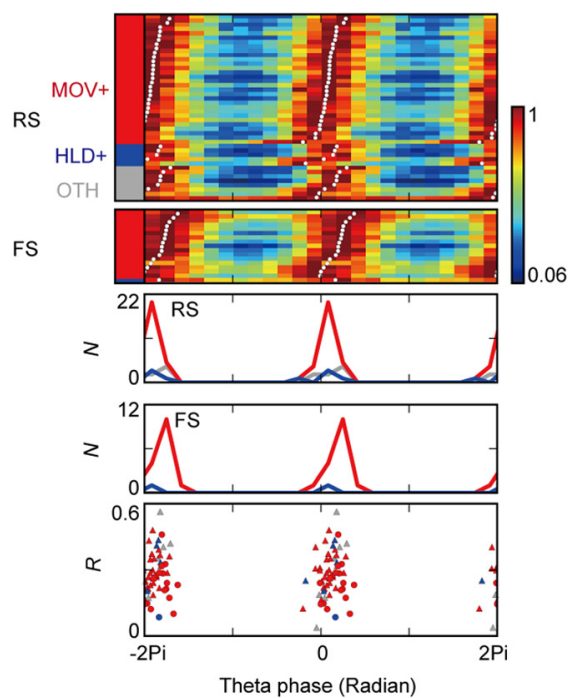

C

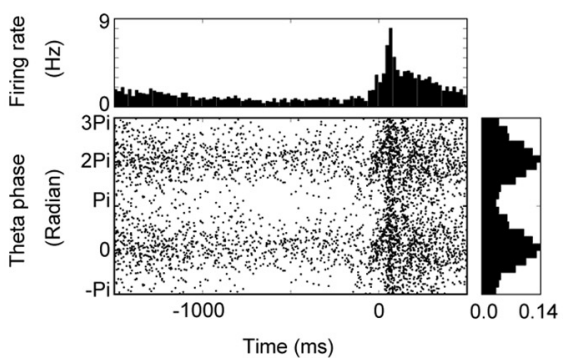

B
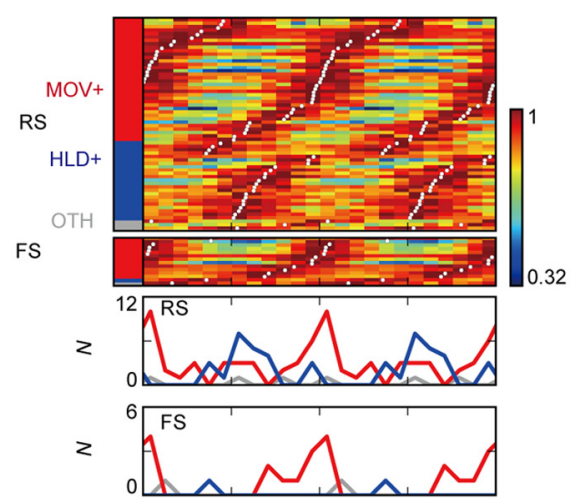

단

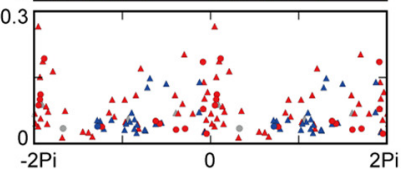

Theta phase (Radian)

D

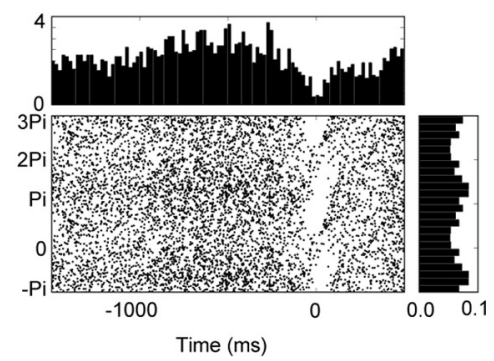

Figure 9. Phase-locking of simultaneously recorded neurons to $\theta$ oscillation. The temporal relationship between neuronal firing and $\theta$ oscillations is shown for the LFP recorded at the depth of $450 \mu \mathrm{m}$. $A$, Density functions of the spiking phases with respect to $\theta$ oscillation are shown for $\mathrm{MOV}^{+}{ }^{+}$(red), $\mathrm{HLD}^{+}$(blue), and $\mathrm{OTH}$ (gray) RS neurons and putative FS interneurons obtained from the superficial layer by the tetrode sites of a silicon probe (top). Among the $46 \mathrm{RS}$ neurons and $17 \mathrm{FS}$ neurons recorded in the superficial layer, $43 \mathrm{RS}$ and all $17 \mathrm{FS}$ neurons exhibited significant phase locking to $\theta$ oscillation. Empty circles represent the mean firing phases of the individual neurons. The instantaneous numbers of active neurons are shown for the three categories of RS neurons (middle upper) and putative FS (middle lower) neurons, together with the $R$ values of phase locking (bottom). The color of each curve indicates the corresponding functional class. $\boldsymbol{B}$, Similar quantities are presented for the deep layer. Among the $95 \mathrm{RS}$ neurons and $15 \mathrm{FS}$ neurons recorded in the deep layer, 62 RS and $14 \mathrm{FS}$ neurons exhibited significant phase locking to $\theta$ oscillation. $C, D$, Spike rastergrams in repeated trials are displayed as functions of time and the $\theta$ phase of $L F P$ for a superficial layer movementrelated and a deep layer hold-related pyramidal neuron, respectively. Histograms above and to the right side of the spike raster represent firing activities aligned at the onset of lever pull $(0 \mathrm{~ms})$ and $\theta$-phase distributions of individual spikes (right), respectively.

\section{Function-specific synchronous firing of motor cortex neurons in $\theta$ and $\gamma$ bands}

The juxtacellular recordings enable us to obtain clean spikes from morphologically identified neurons. However, this method allows us to record only a single morphologically identified neuron from a rat. Therefore, to improve the statistics and explore functional implications of $\theta$ and $\gamma$ oscillations, we examined how these oscillations may synchronize the spikes recorded simultaneously from multiple neurons. We sorted spikes of 81 and 115 neurons from the superficial and deep cortical layers of 15 rats, respectively. These neurons were classified into RS neurons (68 and 102 for superficial and deep layers, respectively) and putative FS interneurons ( 13 for each layer) according to the width of their spikes (Isomura et al., 2009). Namely, we constructed the distribution of the spike width and fitted it with a mixture of two Gaussian distributions that minimizes the classification error (Fig. 8A). Neurons with narrower or broader spikes were classified as putative FS or regular spiking (RS) neurons, respectively.
The known population ratio of various neuron types in the cortex suggests that the majority of RS neurons should be pyramidal cell, though they can also contain non-fast spiking interneurons. Conversely, some pyramidal cells are known to discharge thin spikes and may be misclassified as interneurons (Vigneswaran et al., 2011; Suter et al., 2012). The multiunit data from the superficial layer, which was analyzed newly in this study, revealed an interesting tendency that the superficial layer contained an unexpectedly large portion of Movement-related neurons (Fig. $8 B$ ), or $\mathrm{MOV}+$ neurons (Materials and Methods). Thus, as discussed previously (Isomura et al., 2009), an expectation that the superficial and deep layers govern movement preparation and execution, respectively, is unlikely.

Thus-obtained RS and putative FS neurons exhibited similar tendency of phase locking to what we previously showed for juxtacellularly recorded neurons. Among the 46 RS neurons recorded in the superficial layer, 41 (1) and 44 (4) neurons exhibited significant phase locking to slow and fast $\gamma$ oscillations, respectively, where the counts in parentheses show the numbers of neurons phaselocked only to slow or fast $\gamma$. Among 17 putative FS neurons, 16 (0) and 17 (1) neurons showed significant phase locking in slow and fast $\gamma$ bands, respectively. Among the $95 \mathrm{RS}$ neurons recorded in the deep layer, 49 (0) and 91 (42) neurons exhibited significant phase locking to slow and fast $\gamma$ oscillations, respectively. Among 15 putative FS neurons, 14 (0) and 15 (1) neurons showed significant phase locking in slow and fast $\gamma$ bands, respectively. Therefore, 40 superficial-layer and 49 deep-layer pyramidal neurons exhibited phase locking in both $\gamma$ bands, and so did 16 superficial-layer and 14 deep-layer FS neurons. The probability of firing in consecutive $\gamma$ cycles of the LFPs revealed that both RS and putative FS neurons fired near the troughs of $\gamma$ oscillations in both layers (Fig. $8 C-F$ ), implying that neuronal firing is generally phase-locked to the troughs of $\gamma$ oscillation at the depth of a cell body. The phase-locked firing occurred across cell types and functional neuronal subtypes with timing jitters of $\sim \pm 5 \mathrm{~ms}$. Firing rate was correlated with the velocity of lever movement in many Movement-related neurons (Fig. 8G), although the relationship between the two quantities was divergent and heterogeneous. This seems consistent with observations in the primate motor cortex where direction-tuned neurons generally show a complex scaling of firing rate with the velocity of arm movement (Churchland and Shenoy, 2007; Hatsopoulos and Suminski, 2011). Importantly, the periods of enhanced $\mathrm{MOV}^{+}$(RS and putative FS) or $\mathrm{HLD}^{+}(\mathrm{RS})$ activation coincide with those of strong fast (Spearman's $\rho=0.94, p<0.01$ ) or slow $\gamma$ oscillations $(\rho=0.66, p<0.01$ ), respectively (compare Fig. $8 H$ and Fig. $1 G$ ). 


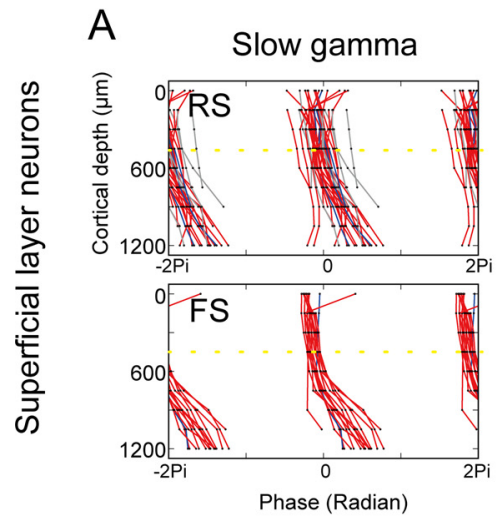

B
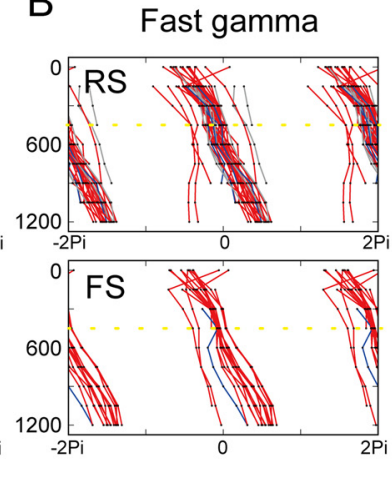

E

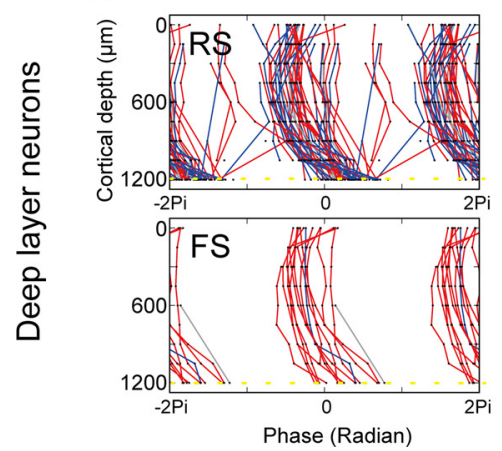

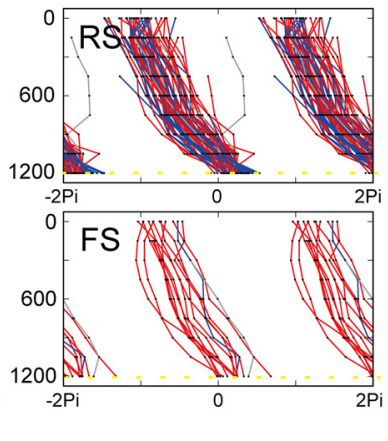

C Theta
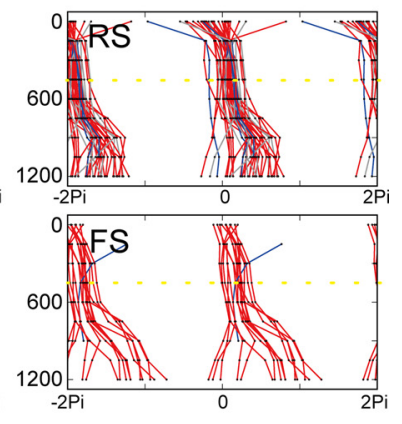

F

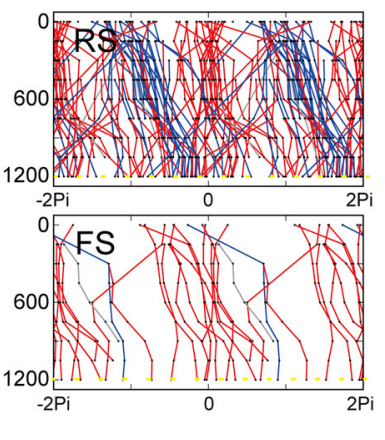

Figure 10. The spiking phases of RS neurons and FS neurons recorded with the tetrode sites of a silicon probe. The LFP oscillations were recorded at different cortical depths with the eight electrode sites and deep tetrode site of the silicon probe. $\boldsymbol{A}-\boldsymbol{C}$, The spike phases with respect to slow $\gamma$, fast $\gamma$, and $\theta$ oscillations, respectively, are shown for RS and FS neurons recorded with a tetrode site placed at the superficial layer. $\mathbf{D}-\boldsymbol{F}$, Similar spike phases are shown for neurons recorded with a tetrode site placed at the deep layer. Red represents MOV ${ }^{+}$neurons; blue represents HLD ${ }^{+}$ neurons; gray represents $0 \mathrm{TH}$ neurons.

The surprising coordination of cell assembles in relation to $\theta$ oscillation is now clearer than before. Most neurons in the superficial layer, where the majority subgroup comprises $\mathrm{MOV}^{+}$neurons, fired synchronously at the troughs of $\theta$ oscillation of LFP regardless of their functional subtypes (Fig. 9A). In striking contrast, neurons with different functional subtypes fired at different phases of $\theta$ oscillation in the deep layer: $\mathrm{MOV}^{+}$neurons fired either near the troughs, as in the superficial layer, or the late portion of descending $\theta$ phases, whereas $\mathrm{HLD}^{+}$neurons fired near the midpoints of descending phases (Fig. 9B). The distributions of the firing phase were significantly different between the two functional subtypes (mean \pm SD: $-16.2 \pm 84.1$ degrees for $\mathrm{MOV}^{+}$and $111.5 \pm 82.5$ degrees for $\mathrm{HLD}^{+} ; p<0.01$ by Watson's $\mathrm{U}^{2}$ test). We asked whether motor cortex neurons exhibit $\theta$-phase precession of spiking as observed in the hippocampus (O'Keefe and Recce, 1993), entorhinal cortex (Hafting et al., 2008), and striatum (van der Meer and Redish, 2011). However, we did not find evidence suggesting phase precession during movement execution (Fig. 9C), nor could we during lever hold (Fig. 9D), from the time evolution of spiking phases of single neurons. We note that the steep slopes of spiking phases near the time origin reflect the phase locking between $\theta$ oscillation and movement onsets (Fig. 2B).

Figure $10 A, B, D, E$ summarizes the relative phases of spikes to slow and fast $\gamma$ oscillations, respectively, of LFPs measured at different depths for all the neurons sorted in the superficial and deep layers. The spiking phases are advanced with the depth of recordings in fast and slow $\gamma$ oscillations because the phases of the LFP oscillations are also advanced. The $\theta$ phases of spiking do not significantly depend on the cell types and functional neuronal subtypes of superficial layer neurons (Fig. 10C), whereas the phases distribute broadly and segregate the two functional subtypes, $\mathrm{MOV}^{+}$and $\mathrm{HLD}^{+}$, in the deep layer (Fig. $10 \mathrm{~F}$ ).

Because the LFP oscillations were mostly accounted for by one or two principal components for any frequency band (Fig. 3), we investigated whether the phase-locked spiking of RS and putative FS neurons in the superficial and deep layers exhibit some preferences to the depth recordings of LFPs. We calculated the resultant length $R$ of the circular distributions of spiking phases for each neuron with respect to the LFP oscillations recorded at different depths. The superficial-layer neurons in general phaselocked most strongly to the LFPs recorded at the depth of $450 \mu \mathrm{m}$ (Fig. 11A-C), and deep-layer FS interneurons showed a similar tendency in the slow $\gamma$ band (Fig. 11D). However, this tendency is weaker or invisible in many deep-layer RS neurons. Moreover, in fast $\gamma$ band, deep-layer RS and FS neurons most strongly phaselocked to the LFP recorded in the deep layers (Fig. 11E). Compared with superficial-layer neurons, deep-layer neurons showed complex depth preferences for $\theta$ oscillation (Fig. $11 F$ ).

\section{Discussion}

\section{A conserved oscillation code between hippocampus and motor cortex}

We have shown that slow and fast $\gamma$ oscillations switch their dominance in different phases of a sequential motor behavior and entrain spiking activity of the majority of task-related neurons across cortical layers. Moreover, $\theta$ oscillations hierarchically coordinate these slow and fast $\gamma$ oscillations, and neurons coding for different stages of sequential motor behavior fire in distinct phases of $\theta$ oscillations, particularly in the deep layer. We sche- 
A

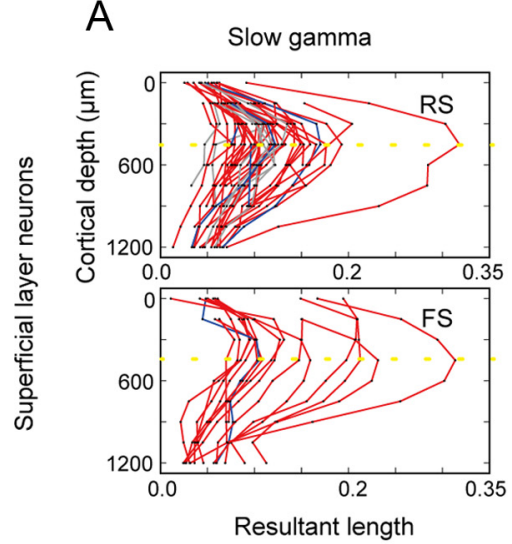

D

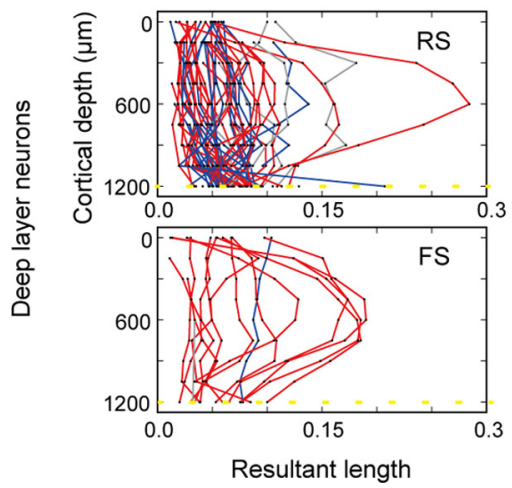

B

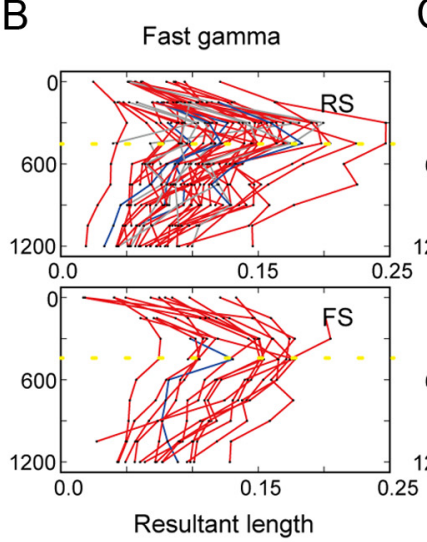

E

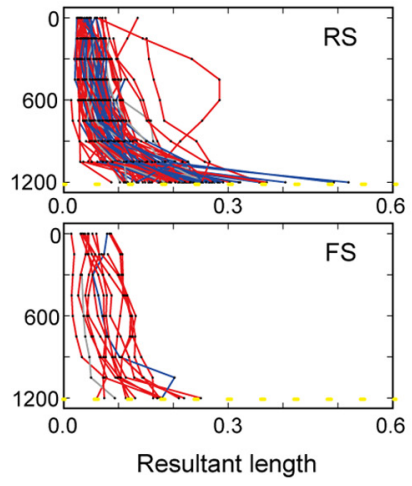

C
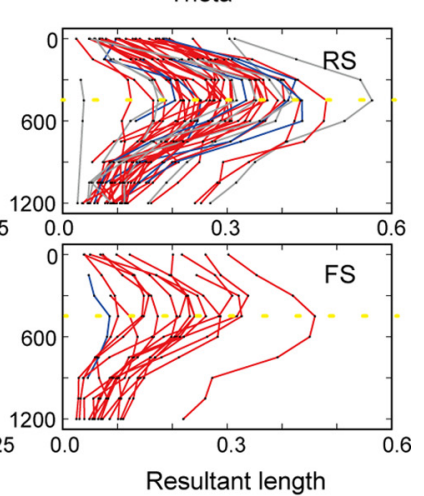

$\mathrm{F}$

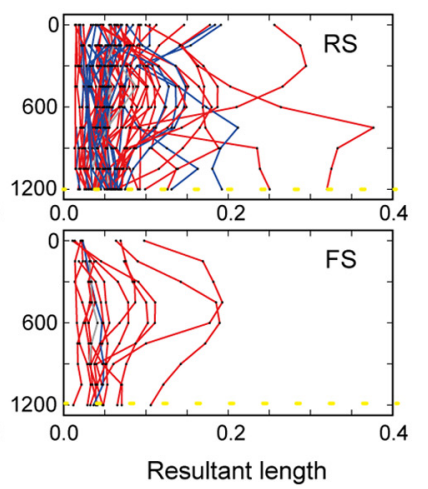

Figure 11. The degrees of phase locking to LFP oscillations at different depths. $\boldsymbol{A}-\boldsymbol{C}$, The depth dependences of the degree of phase locking are shown for slow $\gamma$, fast $\gamma$, and $\theta$ oscillations for RS and FS neurons recorded with a tetrode site placed at the superficial layer: red represents $\mathrm{MOV}^{+}$; blue represents $\mathrm{HLD}^{+}$; gray represents $0 \mathrm{TH}$; yellow represents tetrode position. $\mathbf{D}-\boldsymbol{F}$, The depth dependences are shown for neurons recorded with a tetrode site placed at the deep layer.

matically summarize our findings in Figure 12. $\theta$-nested slow and fast $\gamma$ oscillations are well established in the rat hippocampus (Colgin et al., 2009; Lisman and Jensen, 2013). The striking similarity between motor cortex and hippocampus was previously unknown.

Does the modulation of $4-10 \mathrm{~Hz}$ power around movement onset represents genuine $\theta$ oscillations or merely slow motorrelated potentials? The LFP signal should show a clear rhythmic pattern in the former case, whereas the signal should exhibit a positive or negative peak around movement onsets in the latter case. Several pieces of evidence support the former. First, the power of $\theta$ oscillation was strong during the lever hold and was reduced, rather than enhanced, around movement onset (Fig. $1 G, I)$. Second, the raw trace of LFP exhibited a clear tendency of $\theta$ band oscillation from the lever hold to the pull onset (Fig. 2A). Third, neuronal firing patterns showed characteristic features of phase locking to $\theta$ band oscillation for the entire lever hold period $(>1 \mathrm{~s})$, which should correspond to at least 7 or $8 \theta$ cycles (Fig. $9 C, D)$.

Anatomically, microcircuits of hippocampus and neocortex share several features (Grillner et al., 2005; Shepherd, 2011), such as dense recurrent connections in neocortical L2-L3 and hippocampal CA3 and their primarily feedforward projections to L5 and CA1, respectively (Kaneko et al., 2000; Weiler et al., 2008; Hooks et al., 2011). Physiologically, both L2-L3 (Isomura et al., 2009; de Kock and Sakmann, 2009) and CA3 (Leutgeb et al., 2004; Karlsson and Frank, 2008) networks exhibit sparse firing crucial for efficient information storage (Amit and Treves, 1989). Both hippocampal-based spatial exploration and neocortical-based motor behavior require the ability to store, recall, and process sequence information. Therefore, the parallels in the functional organization of activity described here are sensible from the standpoint of behavior.

Hippocampal and motor cortical circuits also exhibit interesting differences that may specifically relate to the requirements of motor behavior. Whereas the majority of CA1 neurons are phaselocked to either slow or fast $\gamma$ oscillations (Colgin et al., 2009), the majority of motor cortex neurons are phase-locked to both oscillations. Neuronal activities also do not show $\theta$ phase precession in motor cortex. These physiological differences may reflect the functional difference between the hippocampal circuit specialized for memory processing (Nakashiba et al., 2012) and the "general-purpose neocortical circuit," such as transient memory storage and its readout (Maass et al., 2002; Jaeger and Haas, 2004; Sussillo and Abbott, 2009). In addition, such differences may reflect the structural difference that the 6-layer cortical microcircuit has more complicated input and output pathways than the 3-layer hippocampal microcircuit.

\section{Relevance of $\boldsymbol{\theta}-\boldsymbol{\gamma}$ oscillations to motor behavior}

Fast and slow $\gamma$ oscillations are dominant during the execution of lever movements and during lever hold or preparatory periods, respectively. Because posture and movement were suggested to have specialized control processes in monkey motor cortex (Kurtzer et al., 2005), the shift in the prominence of $\gamma$ frequencies may reflect a shift in the distinct control processes for lever hold and movement in the motor cortex. Alternatively, network oscillations may support transient communication across brain struc- 
tures (Canolty and Knight, 2010; Fujisawa and Buzsáki, 2011) necessary at various stages of motor behavior. As in sensory cortex (Womelsdorf and Fries, 2007; Gregoriou et al., 2009), $\gamma$ oscillations may also mediate motor attention that links voluntary effort, information from biological sensors, and movement execution.

Movement-related shifts in oscillation frequency have been known also in primates. The motor cortex of humans shows a transient increase in $\gamma$ band $(80 \mathrm{~Hz})$ activity around the time of movement (Crone et al., 1998; Gaetz et al., 2010; Yanagisawa et al., 2012), as in the high- $\gamma$ activity reported here, whereas $\mu(8-14 \mathrm{~Hz})$ and $\beta(15-30$ $\mathrm{Hz}$ ) oscillations are prominent during movement preparation and suppressed during movement execution (Miller et al., 2012). Similar changes in $\beta$ power are also known in monkeys (Baker et al., 1997; Reimer and Hatsopoulos, 2010). The transient suppression of $\beta$ oscillation is followed by a postmovement rebound exceeding the resting level, whereas $\mu$ oscillation displays little postmovement rebound (Jurkiewicz et al., 2006; van Wijk et al., 2012). These properties of $\mu / \beta$ band activities in the primate motor cortex resemble those of $\theta /$ slow $\gamma$ activities in the rat motor cortex, suggesting that they may play similar functional roles in both species. However, the functional relevance of $\beta$ band activity remains controversial (Engel and Fries, 2010).

In the rat hippocampus, slow $\gamma$ oscillations were suggested to link CA1 with CA3 for retrieving information on previously stored memories (Colgin et al., 2009) and are especially strong in the superficial layer of motor cortex (Fig. 1D). Therefore, slow $\gamma$ band may be related to certain computation by recurrent neural networks, such as memory retrieval or sequence generation. On the other hand, fast $\gamma$ oscillations were suggested to link CA1 with the entorhinal cortex for loading new sensory information (Colgin et al., 2009) and are strong in motor cortex during movement execution. Fast $\gamma$ band may link L5 of motor cortex with spinal cord, similarly to sensory loading in hippocampus, except in this case it could represent the unloading of analog information for patterned motor output (Fukai, 2000). Because the power of fast $\gamma$ oscillations is not significantly different between the superficial and deep layers (Fig. 1D), these oscillations may also link the L2-L3 network to L5 for movement execution via feedforward projections from L2-L3 to L5 (Kaneko et al., 2000; Weiler et al., 2008; Hooks et al., 2011).

\section{Cortical oscillations for microcircuit selection}

Because slow $\gamma$ oscillations (more precisely, oscillations at $\sim 28$ $\mathrm{Hz}$ ) were induced pharmacologically in a slice preparation of the rat motor cortex (Yamawaki et al., 2008), mechanisms underlying synchronized $\gamma$ oscillations are likely to be cortical. They involve gap-junction-connected local networks of FS interneurons and recurrent networks of pyramidal neurons and FS interneurons (Wang, 2010). In the subiculum, FS interneuron networks were suggested for fast $\gamma$ oscillations, whereas pyramidal-interneuron networks were suggested for slow $\gamma$ oscil- lations (Jackson et al., 2011). By contrast, in the rat auditory cortex, the former was suggested for slow $\gamma$ in L2-L3 and the latter for fast $\gamma$ in L4 (Ainsworth et al., 2011). Fast rhythmic bursting pyramidal neurons also promote $\gamma$ band synchronous firing of thalamocortical networks (Cardin et al., 2005). The generation mechanism of slow and fast $\gamma$ oscillations remains to be clarified in motor cortex.

The laminar pattern of slow and fast $\gamma$ oscillations was explained by two principal components, suggesting that separate cellular populations are activated along the array of laminar electrodes. In view of anatomical connections, the first principal component may reflect input from the cerebellum and/or the basal ganglia (via the thalamus) to the superficial layers (Kuramoto et al., 2009) or recurrent input within the layers (Weiler et al., 2008; Hooks et al., 2011). In contrast, the second principal component may represent excitatory interlaminar projections from L2-L3 to L5 (Kaneko et al., 2000; Weiler et al., 2008; Hooks et al., 2011). In addition, interlaminar inhibitory projections from L5 to L2-L3 (Adesnik and Scanziani, 2010; Kätzel et al., 2011; Apicella et al., 2012; Bastos et al., 2012) and corticocortical input to motor cortex may also contribute to the laminar CSD pattern.

The origins of $\theta$ oscillation remain to be clarified. Because the laminar LFPs do not display a clear reversal (Fig. $3 E$ ), $\theta$ oscillation likely contains volume conduction from distant regions, such as the hippocampus (Sirota et al., 2008). Because $\theta$-phase-locked firing is functional subtype- and laminar-specific, $\theta$ oscillation may also arise from local circuit mechanisms, such as a subtype of interneurons (Beierlein et al., 2000; Blatow et al., 2003; Ma et al., 
2006; Sohal et al., 2009). Moreover, $\theta$ oscillation may reflect thalamocortical inputs projecting to the superficial to layer $5 \mathrm{~A}$ of rat motor cortex (Hooks et al., 2013; Kaneko, 2013) because some thalamic neurons exhibit $\theta$ rhythmic firing (Tsanov et al., 2011). Related to this, in the rat sensorimotor cortex, $\alpha$ oscillation $(8-13$ $\mathrm{Hz}$ ), which may correspond to $\theta$ oscillation here, is likely driven by the thalamus (Hughes and Crunelli, 2005), or specifically by thalamocortical bursting neurons (Lorincz et al., 2009). These neurons fire at the troughs and peaks of $\alpha$ oscillation, and might cause the antiphase firing of deep-layer $\mathrm{MOV}^{+}$and $\mathrm{HLD}^{+}$neurons. Alternatively, lateral inhibition between these neuron subtypes may cause such firing.

We have shown that slow and fast $\gamma$ oscillations entrain neuronal firing and are hierarchically coordinated by $\theta$ oscillations in motor cortex. These oscillations may help to select the appropriate microcircuit channels (e.g., layers and cell types) for information transmission driving motor planning and output. We suggest that the coupled oscillations provide a common language for cortical circuit functions.

\section{References}

Adesnik H, Scanziani M (2010) Lateral competition for cortical space by layer-specific horizontal circuits. Nature 464:1155-1160. CrossRef Medline

Ainsworth M, Lee S, Cunningham MO, Roopun AK, Traub RD, Kopell NJ, Whittington MA (2011) Dual $\gamma$ rhythm generators control interlaminar synchrony in auditory cortex. J Neurosci 31:17040-17051. CrossRef Medline

Amit DJ, Treves A (1989) Associative memory neural network with low temporal spiking rates. Proc Natl Acad Sci U S A 86:7871-7875. CrossRef Medline

Apicella AJ, Wickersham IR, Seung HS, Shepherd GM (2012) Laminarly orthogonal excitation of fast spiking and low-threshold-spiking interneurons in mouse motor cortex. J Neurosci 32:7021-7033. CrossRef Medline

Baker SN, Olivier E, Lemon RN (1997) Coherent oscillations in monkey motor cortex and hand muscle EMG show task-dependent modulation. J Physiol 501:225-241. CrossRef Medline

Bastos AM, Usrey WM, Adams RA, Mangun GR, Fries P, Friston KJ (2012) Canonical microcircuits for predictive coding. Neuron 76:695-711. CrossRef Medline

Beierlein M, Gibson JR, Connors BW (2000) A network of electrically coupled interneurons drives synchronized inhibition in neocortex. Nat Neurosci 3:904-910. CrossRef Medline

Blatow M, Rozov A, Katona I, Hormuzdi SG, Meyer AH, Whittington MA, Caputi A, Monyer H (2003) A novel network of multipolar bursting interneurons generates $\theta$ frequency oscillations in neocortex. Neuron 38 : 805-817. CrossRef Medline

Bragin A, Jandó G, Nádasdy Z, Hetke J, Wise K, Buzsáki G (1995) $\gamma(40-100$ $\mathrm{Hz}$ ) oscillation in the hippocampus of the behaving rat. J Neurosci 15:4760. Medline

Canolty RT, Knight RT (2010) The functional role of cross-frequency coupling. Trends Cogn Sci 14:506-515. CrossRef Medline

Cardin JA, Palmer LA, Contreras D (2005) Stimulus-dependent $\gamma(30-50$ $\mathrm{Hz}$ ) oscillations in simple and complex fast rhythmic bursting cells in primary visual cortex. J Neurosci 25:5339-5350. CrossRef Medline

Cho RH, Segawa S, Okamoto K, Mizuno A, Kaneko T (2004) Intracellularly labeled pyramidal neurons in the cortical areas projecting to the spinal cord: II. Intra- and juxta-columnar projection of pyramidal neurons to corticospinal neurons. Neurosci Res 50:395-410. CrossRef Medline

Churchland MM, Shenoy KV (2007) Temporal complexity and heterogeneity of single-neuron activity in premotor and motor cortex. J Neurophysiol 97:4235-4257. CrossRef Medline

Colgin LL, Denninger T, Fyhn M, Hafting T, Bonnevie T, Jensen O, Moser MB, Moser EI (2009) Frequency of $\gamma$ oscillations routes flow of information in the hippocampus. Nature 462:353-357. CrossRef Medline

Crone NE, Miglioretti DL, Gordon B, Lesser RP (1998) Functional mapping of human sensorimotor cortex with electrocorticographic spectral analysis: II. Event-related synchronization in the $\gamma$ band. Brain 121:2301-2315. CrossRef Medline de Kock CP, Sakmann B (2009) Spiking in primary somatosensory cortex during natural whisking in awake head-restrained rats is cell-type specific. Proc Natl Acad Sci U S A 106:16446-16450. CrossRef Medline

Donoghue JP, Sanes JN, Hatsopoulos NG, Gaál G (1998) Neural discharge and local field potential oscillations in primate motor cortex during voluntary movements. J Neurophysiol 79:159-173. Medline

Engel AK, Fries P (2010) $\beta$ band oscillations: signalling the status quo? Curr Opin Neurobiol 20:156-165. CrossRef Medline

Fujisawa S, Buzsáki G (2011) A $4 \mathrm{~Hz}$ oscillation adaptively synchronizes prefrontal, VTA, and hippocampal activities. Neuron 72:153-165. CrossRef Medline

Fukai T (2000) Neuronal communication within synchronous $\gamma$ oscillations. Neuroreport 11:3457-3460. CrossRef Medline

Gaetz W, Macdonald M, Cheyne D, Snead OC (2010) Neuromagnetic imaging of movement-related cortical oscillations in children and adults: age predicts post-movement $\beta$ rebound. Neuroimage 51:792-807. CrossRef Medline

Gregoriou GG, Gotts SJ, Zhou H, Desimone R (2009) Long-range neural coupling through synchronization with attention. Prog Brain Res 176:3545. CrossRef Medline

Grillner S, Markram H, De Schutter E, Silberberg G, LeBeau FE (2005) Microcircuits in action: from CPGs to neocortex. Trends Neurosci 28:525533. CrossRef Medline

Hafting T, Fyhn M, Bonnevie T, Moser MB, Moser EI (2008) Hippocampusindependent phase precession in entorhinal grid cells. Nature 453:1248 1252. CrossRef Medline

Hatsopoulos NG, Suminski AJ (2011) Sensing with the motor cortex. Neuron 72:477-487. CrossRef Medline

Hooks BM, Hires SA, Zhang YX, Huber D, Petreanu L, Svoboda K, Shepherd GM (2011) Laminar analysis of excitatory local circuits in vibrissal motor and sensory cortical areas. PLoS Biol 9:e1000572. CrossRef Medline

Hooks BM, Mao T, Gutnisky DA, Yamawaki N, Svoboda K, Shepherd GM (2013) Organization of cortical and thalamic input to pyramidal neurons in mouse motor cortex. J Neurosci 33:748-760. CrossRef Medline

Hughes SW, Crunelli V (2005) Thalamic mechanisms of EEG $\alpha$ rhythms and their pathological implications. Neuroscientist 11:357-372. CrossRef Medline

Isomura Y, Harukuni R, Takekawa T, Aizawa H, Fukai T (2009) Microcircuitry coordination of cortical motor information in self-initiation of voluntary movements. Nat Neurosci 12:1586-1593. CrossRef Medline

Jackson J, Goutagny R, Williams S (2011) Fast and slow $\gamma$ rhythms are intrinsically and independently generated in the subiculum. J Neurosci 31: 12104-12117. CrossRef Medline

Jaeger H, Haas H (2004) Harnessing nonlinearity: predicting chaotic systems and saving energy in wireless communication. Science 304:78-80. CrossRef Medline

Jurkiewicz MT, Gaetz WC, Bostan AC, Cheyne D (2006) Post-movement $\beta$ rebound is generated in motor cortex: evidence from neuromagnetic recordings. Neuroimage 32:1281-1289. CrossRef Medline

Jutras MJ, Fries P, Buffalo EA (2009) $\gamma$ band synchronization in the macaque hippocampus and memory formation. J Neurosci 29:12521-12531. CrossRef Medline

Kaneko T (2013) Local connections of excitatory neurons in motorassociated cortical areas of the rat. Front Neural Circuits 7:75. CrossRef Medline

Kaneko T, Cho R, Li Y, Nomura S, Mizuno N (2000) Predominant information transfer from layer III pyramidal neurons to corticospinal neurons. J Comp Neurol 423:52-65. CrossRef Medline

Karlsson MP, Frank LM (2008) Network dynamics underlying the formation of sparse, informative representations in the hippocampus. J Neurosci 28:14271-14281. CrossRef Medline

Kätzel D, Zemelman BV, Buetfering C, Wölfel M, Miesenböck G (2011) The columnar and laminar organization of inhibitory connections to neocortical excitatory cells. Nat Neurosci 14:100-107. CrossRef Medline

Kuramoto E, Furuta T, Nakamura KC, Unzai T, Hioki H, Kaneko T (2009) Two types of thalamocortical projections from the motor thalamic nuclei of the rat: a single neuron-tracing study using viral vectors. Cereb Cortex 19:2065-2077. CrossRef Medline

Kurtzer I, Herter TM, Scott SH (2005) Random change in cortical load representation suggests distinct control of posture and movement. Nat Neurosci 8:498-504. CrossRef Medline

Le Van Quyen M, Staba R, Bragin A, Dickson C, Valderrama M, Fried I, Engel 
J (2010) Large-scale microelectrode recordings of high-frequency $\gamma$ oscillations in human cortex during sleep. J Neurosci 30:7770-7782. CrossRef Medline

Lee D (2003) Coherent oscillations in neuronal activity of the supplementary motor area during a visuomotor task. J Neurosci 23:6798-6809. Medline

Leutgeb S, Leutgeb JK, Treves A, Moser MB, Moser EI (2004) Distinct ensemble codes in hippocampal areas CA3 and CA1. Science 305:12951298. CrossRef Medline

Lisman J, Redish AD (2009) Prediction, sequences and the hippocampus. Philos Trans R Soc Lond B Biol Sci 364:1193-1201. CrossRef Medline

Lisman JE, Jensen O (2013) The $\theta-\gamma$ neural code. Neuron 77:1002-1016. CrossRef Medline

Lorincz ML, Kékesi KA, Juhász G, Crunelli V, Hughes SW (2009) Temporal framing of thalamic relay-mode firing by phasic inhibition during the $\alpha$ rhythm. Neuron 63:683-696. CrossRef Medline

Maass W, Natschläger T, Markram H (2002) Real-time computing without stable states: a new framework for neural computation based on perturbations. Neural Comput 14:2531-2560. CrossRef Medline

Ma Y, Hu H, Berrebi AS, Mathers PH, Agmon A (2006) Distinct subtypes of somatostatin-containing neocortical interneurons revealed in transgenic mice. J Neurosci 26:5069-5082. CrossRef Medline

Miller KJ, Hermes D, Honey CJ, Hebb AO, Ramsey NF, Knight RT, Ojemann JG, Fetz EE (2012) Human motor cortical activity is selectively phaseentrained on underlying rhythms. PLoS Comput Biol 8: e1002655. CrossRef Medline

Murthy VN, Fetz EE (1996) Synchronization of neurons during local field potential oscillations in sensorimotor cortex of awake monkeys. J Neurophysiol 76:3968-3982. Medline

Nakashiba T, Cushman JD, Pelkey KA, Renaudineau S, Buhl DL, McHugh TJ, Rodriguez Barrera V, Chittajallu R, Iwamoto KS, McBain CJ, Fanselow MS, Tonegawa S (2012) Young dentate granule cells mediate pattern separation, whereas old granule cells facilitate pattern completion. Cell 149:188-201. CrossRef Medline

O'Keefe J, Recce ML (1993) Phase relationship between hippocampal place units and the EEG theta rhythm. Hippocampus 3:317-330. CrossRef Medline

Pesaran B, Pezaris JS, Sahani M, Mitra PP, Andersen RA (2002) Temporal structure in neuronal activity during working memory in macaque parietal cortex. Nat Neurosci 5:805-811. CrossRef Medline

Quilichini P, Sirota A, Buzsáki G (2010) Intrinsic circuit organization and $\theta-\gamma$ oscillation dynamics in the entorhinal cortex of the rat. J Neurosci 30:11128-11142. CrossRef Medline

Rahman J, Berger T (2011) Persistent activity in layer 5 pyramidal neurons following cholinergic activation of mouse primary cortices. Eur J Neurosci 34:22-30. CrossRef Medline

Reimer J, Hatsopoulos NG (2010) Periodicity and evoked responses in motor cortex. J Neurosci 30:11506-11515. CrossRef Medline

Sanes JN, Donoghue JP (1993) Oscillations in local field potentials of the primate motor cortex during voluntary movement. Proc Natl Acad Sci U S A 90:4470-4474. CrossRef Medline

Shepherd G (2011) The microcircuit concept applied to cortical evolution: from three-layer to six-layer cortex. Front Neuroanat 5:30. CrossRef Medline

Sirota A, Montgomery S, Fujisawa S, Isomura Y, Zugaro M, Buzsáki G (2008) Entrainment of neocortical neurons and $\gamma$ oscillations by the hippocampal $\theta$ rhythm. Neuron 60:683-697. CrossRef Medline
Sohal VS, Zhang F, Yizhar O, Deisseroth K (2009) Parvalbumin neurons and $\gamma$ rhythms enhance cortical circuit performance. Nature 459:698702. CrossRef Medline

Sukov W, Barth DS (1998) Three-dimensional analysis of spontaneous and thalamically evoked $\gamma$ oscillations in auditory cortex. J Neurophysiol 79: 2875-2884. Medline

Sussillo D, Abbott LF (2009) Generating coherent patterns of activity from chaotic neural networks. Neuron 63:544-557. CrossRef Medline

Suter BA, Migliore M, Shepherd GM (2012) Intrinsic electrophysiology of mouse corticospinal neurons: a class-specific triad of spike-related properties. Cereb Cortex 23:1965-1977. CrossRef Medline

Szurhaj W, Derambure P (2006) Intracerebral study of $\gamma$ oscillations in the human sensorimotor cortex. Prog Brain Res 159:297-310. CrossRef Medline

Takekawa T, Isomura Y, Fukai T (2012) Spike-sorting of heterogeneous neuron types by multimodality-weighted PCA and explicit robust variational Bayes. Front Neuroinform 6:5. CrossRef Medline

Thomas JB (1969) An introduction to statistical communication theory. New York: Wiley.

Torrence C, Compo GP (1998) A practical guide to wavelet analysis. Bull Am Meteor Soc 79:61-78. CrossRef

Tsanov M, Chah E, Vann SD, Reilly RB, Erichsen JT, Aggleton JP, O’Mara SM (2011) $\theta$-modulated head direction cells in the rat anterior thalamus. J Neurosci 31:9489-9502. CrossRef Medline

van der Meer MA, Redish AD (2011) $\theta$ phase precession in rat ventral striatum links place and reward information. J Neurosci 31:2843-2854. CrossRef Medline

van der Meer MA, Kalenscher T, Lansink CS, Pennartz CM, Berke JD, Redish $\mathrm{AD}$ (2010) Integrating early results on ventral striatal $\gamma$ oscillations in the rat. Front Neurosci 4:300. CrossRef Medline

van Wijk BC, Beek PJ, Daffertshofer A (2012) Neural synchrony within the motor system: what have we learned so far? Front Hum Neurosci 6:252. CrossRef

Vigneswaran G, Kraskov A, Lemon RN (2011) Large identified pyramidal cells in macaque motor and premotor cortex exhibit "thin spikes": implications for cell type classification. J Neurosci 31:14235-14242. CrossRef Medline

Vinck M, Battaglia FP, Womelsdorf T, Pennartz C (2012) Improved measures of phase-coupling between spikes and the local field potential. J Comput Neurosci 33:53-75. CrossRef Medline

Wang XJ (2010) Neurophysiological and computational principles of cortical rhythms in cognition. Physiol Rev 90:1195-1268. CrossRef Medline

Weiler N, Wood L, Yu J, Solla SA, Shepherd GM (2008) Top-down laminar organization of the excitatory network in motor cortex. Nat Neurosci 11:360-366. CrossRef Medline

Womelsdorf T, Fries P (2007) The role of neuronal synchronization in selective attention. Curr Opin Neurobiol 17:154-160. CrossRef Medline

Yamawaki N, Stanford IM, Hall SD, Woodhall GL (2008) Pharmacologically induced and stimulus evoked rhythmic neuronal oscillatory activity in the primary motor cortex in vitro. Neuroscience 151:386-395. CrossRef Medline

Yanagisawa T, Yamashita O, Hirata M, Kishima H, Saitoh Y, Goto T, Yoshimine T, Kamitani Y (2012) Regulation of motor representation by phase-amplitude coupling in the sensorimotor cortex. J Neurosci 32: 15467-15475. CrossRef Medline 